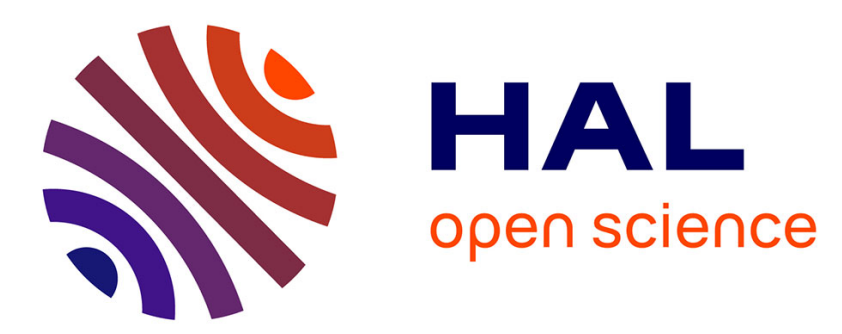

\title{
Stability and optimal forcing analysis of a wind turbine wake: Comparison with POD
}

Giovanni de Cillis, Stefania Cherubini, Onofrio Semeraro, Stefano Leonardi, Pietro de Palma

\section{- To cite this version:}

Giovanni de Cillis, Stefania Cherubini, Onofrio Semeraro, Stefano Leonardi, Pietro de Palma. Stability and optimal forcing analysis of a wind turbine wake: Comparison with POD. Renewable Energy, 2022, 181, pp.765-785. 10.1016/j.renene.2021.09.025 . hal-03498568

\section{HAL Id: hal-03498568 \\ https://hal.science/hal-03498568}

Submitted on 21 Dec 2021

HAL is a multi-disciplinary open access archive for the deposit and dissemination of scientific research documents, whether they are published or not. The documents may come from teaching and research institutions in France or abroad, or from public or private research centers.
L'archive ouverte pluridisciplinaire HAL, est destinée au dépôt et à la diffusion de documents scientifiques de niveau recherche, publiés ou non, émanant des établissements d'enseignement et de recherche français ou étrangers, des laboratoires publics ou privés. 


\title{
Stability and optimal forcing analysis of a wind turbine wake: comparison with POD
}

\author{
Giovanni De Cillis ${ }^{1,2,4, *}$, Stefania Cherubini ${ }^{1}$, Onofrio Semeraro $^{3}$, Stefano Leonardi ${ }^{2}$, \\ Pietro De Palma ${ }^{1}$
}

\begin{abstract}
Understanding the dynamics and generation of coherent structures in wind-turbine wakes is crucial for efficiency improvement of wind farms, which will most probably represent one of the main renewable power generation sources in 2050. In this paper, we investigate the origin of such coherent structures by performing modal and non-modal stability analysis of the mean flow downstream of a wind-turbine rotor. The database consists of large-eddysimulation results. Bi-local linear-stability and optimal-forcing analyses are performed at several wake's cross-sections. Most unstable perturbations are compared with the most energetic coherent structures recovered by the proper orthogonal decomposition (POD) analysis, showing a good agreement close to the rotor. Further downstream, these modes are overtaken by others with wavenumbers departing from those of the main POD modes. However, optimal-forcing analysis shows that asymptotically stable modes can be amplified by more than one order of magnitude via quasi-resonance mechanisms, bypassing the growth of the most unstable modes in the far wake. This suggests that the most energetic structures are originated by modal instabilities, which trigger quasiresonance mechanisms in the far wake, determining the emergence of specific frequencies in the turbulent flow. These findings are crucial for designing efficient control systems to optimize wind farm performance.
\end{abstract}

Keywords: Wind turbine wake, Bi-local stability analysis, Resolvent analysis, Proper Orthogonal Decomposition (POD), Large Eddy Simulation (LES)

\section{Nomenclature}

${ }_{3} \quad \alpha \quad$ Streamwise wavenumber

${ }_{4} \quad \lambda \quad$ Tip-speed ratio

u Nondimensional instantaneous velocity vector

\footnotetext{
*giovanni.decillis@poliba.it

${ }^{1}$ Dipartimento di Meccanica, Matematica e Management, Politecnico di Bari, Via Re David 200, 70125, Bari, Italy

${ }^{2}$ Department of Mechanical Engineering, University of Texas, Dallas, Texas, USA

${ }^{3}$ LIMSI-CNRS, Université de Paris-Saclay, 91440, Orsay, France

${ }^{4}$ European-Mediterranean Centre for Climate Changes, Ocean Predictions and Applications Division, Via Augusto Imperatore 16, 73100, Lecce, Italy
} 
$\mathbf{u}^{\prime} \quad$ Incoherent nondimensional velocity vector

$\Omega \quad$ Nondimensional angular frequency of the rotor

$\omega \quad$ Temporal wavenumber

$\overline{\mathbf{u}} \quad$ Time-averaged nondimensional velocity vector

10 Coherent nondimensional velocity vector

1 D Diameter of the turbine

2 Azimuthal wavenumber

3 Nondimensional pressure

${ }_{4}$ Re Reynolds number

$U_{\infty}$ Dimensional uniform velocity at the inflow

\section{Introduction}

Achieving the Paris climate goals needs a significant acceleration in the exploitation of wind and solar energy, which will most probably represent the main renewable power generation sources in 2050. It is expected that wind power alone would provide about $35 \%$ of the total electricity needs [2], corresponding to a production of about $6000 \mathrm{GW}$, becoming the main power generation source. Such a transformation is possible only through a significant increase of the installed wind power. In fact, the wind-energy share of electricity capacity was about $7,9 \%, 8,5 \%, 9,6 \%$ in $2018,2019,2020$, respectively, corresponding to $564 G W, 622 G W, 733 G W$ [3]. Annual net wind power additions were about $58 G W$ in 2019 and $111 G W$ in 2020. Therefore, about $180 G W$ of average power addition per year would be needed in the future to meet the Paris goal. The global LCOE for onshore wind plants is estimated to fall to $30-50 U S D / M W h$ by 2030 and $20-30 U S D / M W h$ by 2050 [2]. Instead, the LCOE of offshore wind plants should drop to an average of $50-90 U S D / M W h$ by 2030 and $30-70 U S D / M W h$ by $2050[2,4]$. However, the current rate of growth of new wind-energy installed power is not sufficient to meet the Paris climate goals. Due to construction and supply chain delays related to the effects of Covid-19, some Countries have even reduced their capability of capacity addition (China, for instance, installed 30\% less onshore wind capacity in the first half of 2020) [1]. Innovation is needed both for the single turbine technology and for the farm optimization. Concerning the single turbine, the increase of rotor diameters and hub heights will likely lead to an average capacity of $5-6 M W$ by 2035 in the case of onshore applications (from an average of 2.5 MW in 2019); these turbines would have diameters of $160-170 \mathrm{~m}$. In the case of offshore installations, the single wind power would achieve $15-20 \mathrm{MW}$ within 2035 (from the actual average capacity of about $6 \mathrm{MW}$ ), corresponding to diameters of about $230-250 \mathrm{~m}$ [53]. Moreover, the optimization of the farm design and its control with respect to the wind conditions is crucial to guarantee high efficiency, flexibility and security of the power generation, leading us to the topic of the present work, in particular with reference to the turbine wake control. 
Large wind farms are constituted by hundreds of turbines, a great part of which operates in the wake of other turbines. The presence of upwind turbines induces velocity deficit and oscillations in the incoming flow which produce power losses and fatigue blade loading $[7,45]$. For preventing these shortcomings, turbines and farms should be opportunely designed to reduce, when possible, the generation of low-frequency oscillations, such as the wake meandering phenomenon [54], and to accelerate wake recovery. Thus, understanding the dynamics of coherent structures in wind turbine wakes is crucial for the design and efficiency improvement of wind farms. The dynamics of coherent structures in the wake of a wind turbine has been analysed in detail in the literature using the proper orthogonal decomposition (POD) technique $[43,10]$ applied to numerical or experimental data. VerHulst and Meneveau [49] applied this technique to the study of a wind farm. They found that streamwise counter-rotating rolls, which generate ejection and sweep regions, are the dominant coherent structure within the flow. Other authors applied POD analysis to study the wake of a single wind turbine. Bastine et al. $[9,8]$ performed two-dimensional POD analysis using large eddy simulation (LES) of the wake of a wind turbine modeled by an actuator disk, impinged by an incoming turbulent atmospheric boundary layer. A similar two-dimensional POD analysis carried out by Sorensen et al. [44] provided POD modes with distinct spatial structures, such as monopole, dipole, quadrupole and hexapole structures. The most energetic POD modes, displaying a dipole structure around the rotor perimeter, are found to govern the very large scale motion of the wake, often referred to as wake meandering. Debnath et al. [17] used three-dimensional POD analysis to study the dynamics of a single wind-turbine wake. They carried out LESs using the actuator-line technique to simulate the rotor, and the immersed-boundary method to include the tower and nacelle. Several POD modes with different energy content, mostly representing instabilities of the tip vortices, but also the interaction between the rotor wake and the vortex shedding from the turbine tower, have been detected and used to build a reduced order model.

Despite extracting the most energetic flow structures, POD analysis does not provide a deep insight on the physical origin of coherent structures. On the other hand, linear stability analysis of time-averaged mean flows has proven to be a powerful tool able to predict the low-frequency oscillations in statistically stationary turbulent flows. By means of a 'local', one-dimensional stability analysis carried out in the vicinity of the wind-turbine rotor, Iungo et al. [24] found highly unstable eigenmodes with temporal frequency typical of the wake meandering and associated with small azimuthal wavenumbers. A similar one-dimensional analysis has been then performed by Viola et al. [52] adding different eddy viscosity models to take into account the turbulent diffusion of perturbations. This analysis provided eigenspectra with maximum growth rate at temporal and azimuthal wavenumbers corresponding to those typical of the wake meandering phenomenon. The influence of turbulence intensity and blade aerodynamics on the hub-vortex instability frequencies and related flow structures has been studied in references $[6,5]$ using a model mean flow. Viola et al. [51] have carried out a two-dimensional stability analysis in the cross-planes close to the rotor of a model wind turbine immersed in an atmospheric boundary layer, finding once again unstable modes with frequencies typical of the hubvortex instability. More recently, Ferrer et al. [20] used stability and sensitivity analysis on a wall-parallel plane passing through the hub center of a wind turbine rotor to design a passive way to control the primary wake destabilization at low Reynolds number. Focus- 
ing the analysis on a two-dimensional configuration, they showed that adding a localised control force in flow regions identified by the sensitivity analysis can stabilise the wake. Although based on a simplified flow configuration at low Reynolds number, this work indicates that, for controlling the wake, one should modify the velocity gradient close to the turbine in a non trivial way, as predicted by sensitity analysis. Linear stability eigenmodes, together with their adjoint counterparts [21], are thus able to provide valuable information on the shape and location of active or passive means to control the spatial structure, recovery rate, and frequency content of the wake behind a wind turbine.

Despite the importance of linear stability analysis for the identification and control of coherent structures, only a few stability studies, mostly focused on the hub-vortex instability, have been carried out on wind turbine flows. A detailed analysis of the main flow features found by modal and non-modal instability methods, and on their relevance with respect to the coherent structures that populate the turbulent flow, is still lacking in the literature. In fact, apart from the hub-vortex instability recovered by Iungo et al. [24] in the immediate vicinity of the rotor, the correspondence of other linear instabilities to energetic coherent structures extending towards the far wake region has not been investigated yet. Moreover, the possible relevance of non-modal stability mechanisms, able to provide a strong amplification of some particular perturbations, in the dynamics of coherent structures in wind turbine wakes, has not been assessed yet. Non-modal mechanisms such as the amplification of harmonic forcing at particularly receptive frequencies, can have a strong relevance in the development of coherent structures within the wake. In fact, non-modal amplification mechanisms may allow a fast transfer of energy from the mean flow to some waves having particularly receptive frequencies, allowing the displacement of energy among different regions of the wake and strongly affecting wake recovery. The capability of resolvent (optimal forcing) analysis to identify the most energetic flow structures in asymptotically stable flows has been recently proven for different flow configurations [28, 22, 42], and the importance of Reynolds stress modeling and forcing statistics has been highlighted $[34,47]$. However, in our knowledge, resolvent analysis has never been performed on a wind-turbine wake, despite it might potentially provide a deep insight in the origin of the most energetic coherent structures developing into the wake, such as those recovered by POD analysis. Thus, a detailed analysis of the modal and non-modal stability of a wind-turbine wake, framed by a comparison with POD modes, will elucidate the link between the most energetic frequencies and structures developing in the wake and the energy amplification mechanisms originating them, finally providing a profound insight into their control.

Towards this aim, in the present paper, we investigate in detail the modal and nonmodal stability of the turbulent mean flow developing downstream of a wind turbine rotor, computed by LES using the actuator line technique to simulate the rotor. Since stability analysis aims at finding intrinsic oscillations of the flow, which arise even in the absence of ambient turbulence, the flow impinging on the turbine is considered to be laminar and uniform. Two-dimensional linear stability and optimal forcing analyses have been carried out at different cross-flow planes sufficiently far from the rotor, where the non-parallel effect are weak and can be neglected. The frequency content and spatial structure of the most amplified perturbations are then compared with the most energetic coherent structures recovered by POD analysis. This comparison will help understanding the physical mechanisms at the origin of the development of the particular flow structures 
and wavenumbers of the most energetic POD modes.

The paper is structured as follows. We present the numerical simulation in $\S 2$ and the flow setting in $\S 2.1$. The numerical dataset used as a benchmark for the stability analysis is introduced in $\S 3$, and analysed by means of data-driven modal analysis in $\S 3.1$. In $\S 4$, we introduce the linear modelling and the stability tools adopted $(\S 4.1$ and $\S 4.2)$; results are discussed in $\S 4.3$ and $\S 4.4$. The paper finalises with conclusions in $\S 5$.

\section{Methodologies}

The Large Eddy Simulation (LES) approach is employed to compute the dynamics of the wake developing behind a wind turbine. Starting from the Navier-Stokes equations for incompressible flows, the governing equations for the filtered non-dimensional velocity, $\mathbf{u}=(u, v, w)^{T}$, and the pressure, $p$, are obtained as

$$
\begin{gathered}
\frac{\partial \mathbf{u}}{\partial t}=-(\mathbf{u} \cdot \boldsymbol{\nabla}) \mathbf{u}-\nabla p+\frac{1}{R e} \nabla^{2} \mathbf{u}-\boldsymbol{\nabla} \cdot \boldsymbol{\tau}+\mathbf{F}, \\
0=\boldsymbol{\nabla} \cdot \mathbf{u},
\end{gathered}
$$

where $\boldsymbol{\tau}$ represents the sub-grid scale stress tensor, and $\mathbf{F}$ represents the aerodynamic forces exerted by the turbine blades on the fluid modeled by the actuator line method (see [13], for instance). The quantities in Eq. (1) are non-dimensional with respect to the free-stream velocity $U_{\infty}$, the rotor diameter $D$ and the kinematic viscosity of the fluid $\nu$, the Reynolds number being defined as $R e=\frac{U_{\infty} D}{\nu}$. The isotropic part of the subgrid-scale stress tensor $\frac{1}{3} \tau_{i i}$ is included in the modified filtered pressure, so that $p^{*}=p+\frac{1}{3} \tau_{i i}$, while the anisotropic part $\tau_{i j}^{r}$ is modeled using the Smagorinsky model with constant $C_{s}=0.09$. This choice has been widely used in similar test cases and validated against different subgrid scale models, showing a weak dependence of the wake dynamics (see MartinezTossas et al. [31] and Ciri et al. [14, 13]). The governing equations are solved using a second-order-accurate centered finite difference scheme using a staggered Cartesian grid, where streamwise, wall-normal and spanwise directions are indicated by $x, y$ and $z$, respectively. For time integration, a hybrid low-storage third-order-accurate RungeKutta scheme is employed [35].

\subsection{Simulation layout}

The simulation layout is based on the experiments performed by Krogstad \& Eriksen [26], using a turbine model with a three-bladed rotor of diameter $D=0.894 \mathrm{~m}$ and hub height of $0.817 \mathrm{~m}$. With respect to the reference case [26], we consider a setup more similar to that of a realistic wind turbine by extending the domain in the wall-normal direction and by removing the lateral walls with the aim of reducing the blockage effect. The dimensions of the computational domain, in diameter units, are $L_{x}=12.5$, $L_{y}=5$ and $L_{z}=3$, in the streamwise $(x)$, wall-normal $(y)$, and spanwise $(z)$ directions, respectively. As sketched in figure 1, the rotor is located at 4 diameters from the inlet section, where a uniform velocity profile $U_{\infty}=10 \mathrm{~m} / \mathrm{s}$, aligned with the rotor axis, is imposed. A radiative non-reflective boundary condition is employed at the outlet points with convection velocity $C=9 \mathrm{~m} / \mathrm{s}$ [36]. No slip boundary conditions are imposed at 
the bottom wall, whereas free slip is prescribed at the top wall. Periodicity is imposed along the spanwise direction. The Reynolds number is $R e=6.3 \times 10^{5}$. The computational domain is discretized using an uniformly-spaced grid with 2048 and 512 points in the streamwise and spanwise direction, respectively. Along the wall-normal direction $y$, a stretched grid consisting of 512 points is used, with finer (uniform) spacing, equal to $\Delta y=0.006$ diameter units, in the rotor-wake region. We impose a tip-speed ratio $\lambda=3$ following the experimental data reported in Ref. [26]. Thus, the dimensionless angular frequency of the rotor is equal to $\Omega=2 \lambda=6$, corresponding to a Strouhal number $S t_{r}=\Omega / 2 \pi=0.9549$.

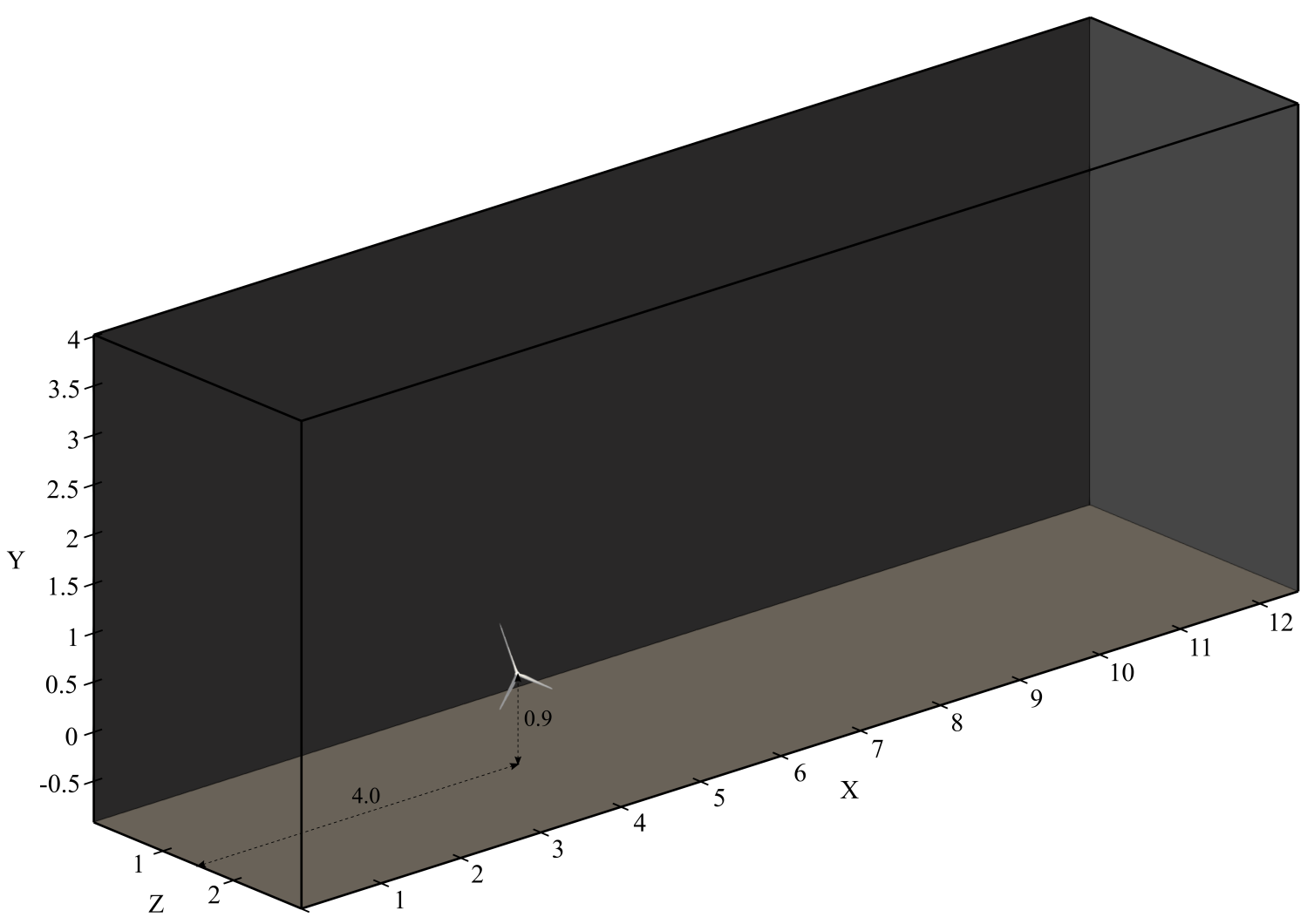

Figure 1: Sketch of the computational domain.

Note that, for validation purposes, we have chosen a configuration in which the Reynolds number is lower than that of utility-scale turbines, and the inflow is laminar. Despite the influence of ambient turbulence on the development of the flow behind the turbine is not negligible $[50,33]$, the absence of inflow turbulence will allow us discriminating the presence of intrinsic stability modes in the turbine wake, from the oscillations that can be already present in the ambient turbulence. In Ref. [16], this numerical layout has been validated by means of a mesh convergence analysis, and used for assessing how the presence of tower and nacelle impacts on wake recovery. The turbine is simulated with and without tower and nacelle and the proper orthogonal decomposition of the wake velocity fields allowed us to isolate the main coherent structures in the two cases. Moreover, we have verified that the structure and frequencies of the main POD modes is not strongly affected by the Reynolds number [15]. One snapshot of the streamwise velocity for each 

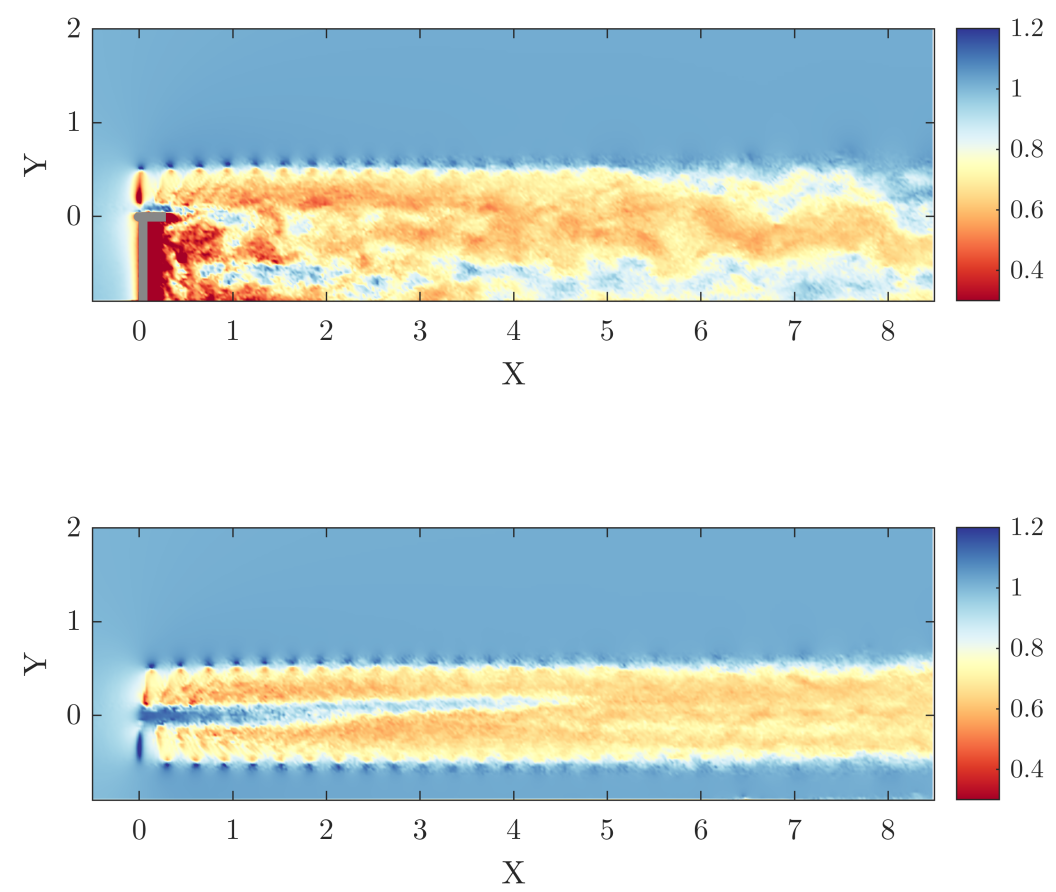

Figure 2: Streamwise velocity contours in the $\mathrm{x}-\mathrm{y}$ plane passing through the rotor axis, for the case with (top frame) and without (bottom frame) tower and nacelle.

case is shown in Fig. 2. POD modes are analysed in terms of their contribution to mean kinetic energy flux within the wake and therefore to wake recovery. In the following, we provide a brief survey of the dataset considered in this investigation and include a summary of the main results which will be considered later as a guideline for the stability analysis

\section{Identification of coherent structures}

The numerical dataset is first analysed using POD in order to identify the coherent structures dominating in the wind-turbine wake. The flow field is decomposed using a set of orthonormal functions $\phi_{j}$, providing a complete basis for each realization of the stochastic process $\boldsymbol{q}(\boldsymbol{x}, t)$. This can be expanded as

$$
\boldsymbol{q}(\boldsymbol{x}, t)=\sum_{j=1}^{\infty} a_{j}(t) \boldsymbol{\phi}_{j}(\boldsymbol{x}),
$$

$a_{j}(t)=\left\langle\boldsymbol{q}(\boldsymbol{x}, t), \boldsymbol{\phi}_{j}(\boldsymbol{x})\right\rangle$ being the time coefficients of the expansion. The spatial modes $\phi(\boldsymbol{x})$ are chosen such that the quotient

$$
\lambda=\frac{E\left\{|\langle\boldsymbol{q}(\boldsymbol{x}, t), \boldsymbol{\phi}(\boldsymbol{x})\rangle|^{2}\right\}}{\langle\boldsymbol{\phi}(\boldsymbol{x}), \boldsymbol{\phi}(\boldsymbol{x})\rangle},
$$

is maximized; in Eq. (3), $\langle\cdot, \cdot\rangle$ denotes the inner product and $E\{\cdot\}$ is the expectation operator. Further details on the POD method can be found in Ref. [10]. In this work, 


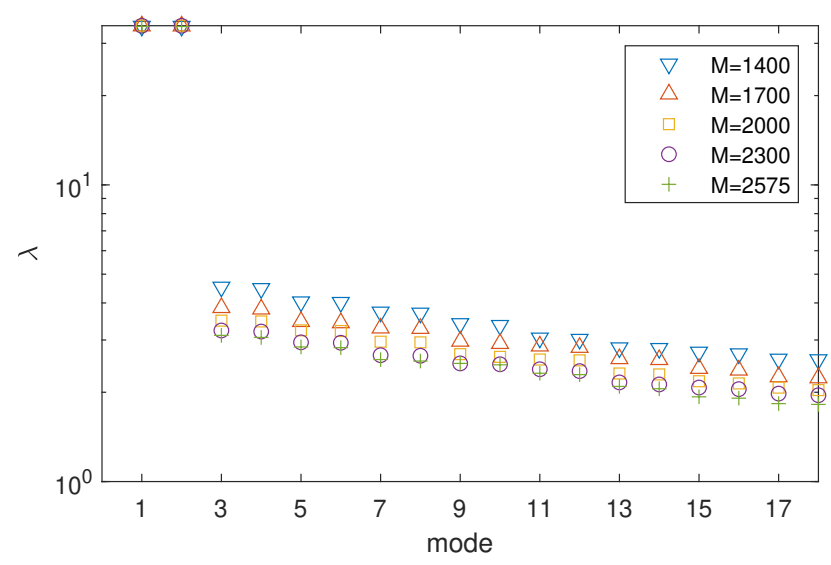

Figure 3: Convergence of the eigenvalues $\lambda$ associated with the first 18 POD modes with respect to the employed number of snapshots M.

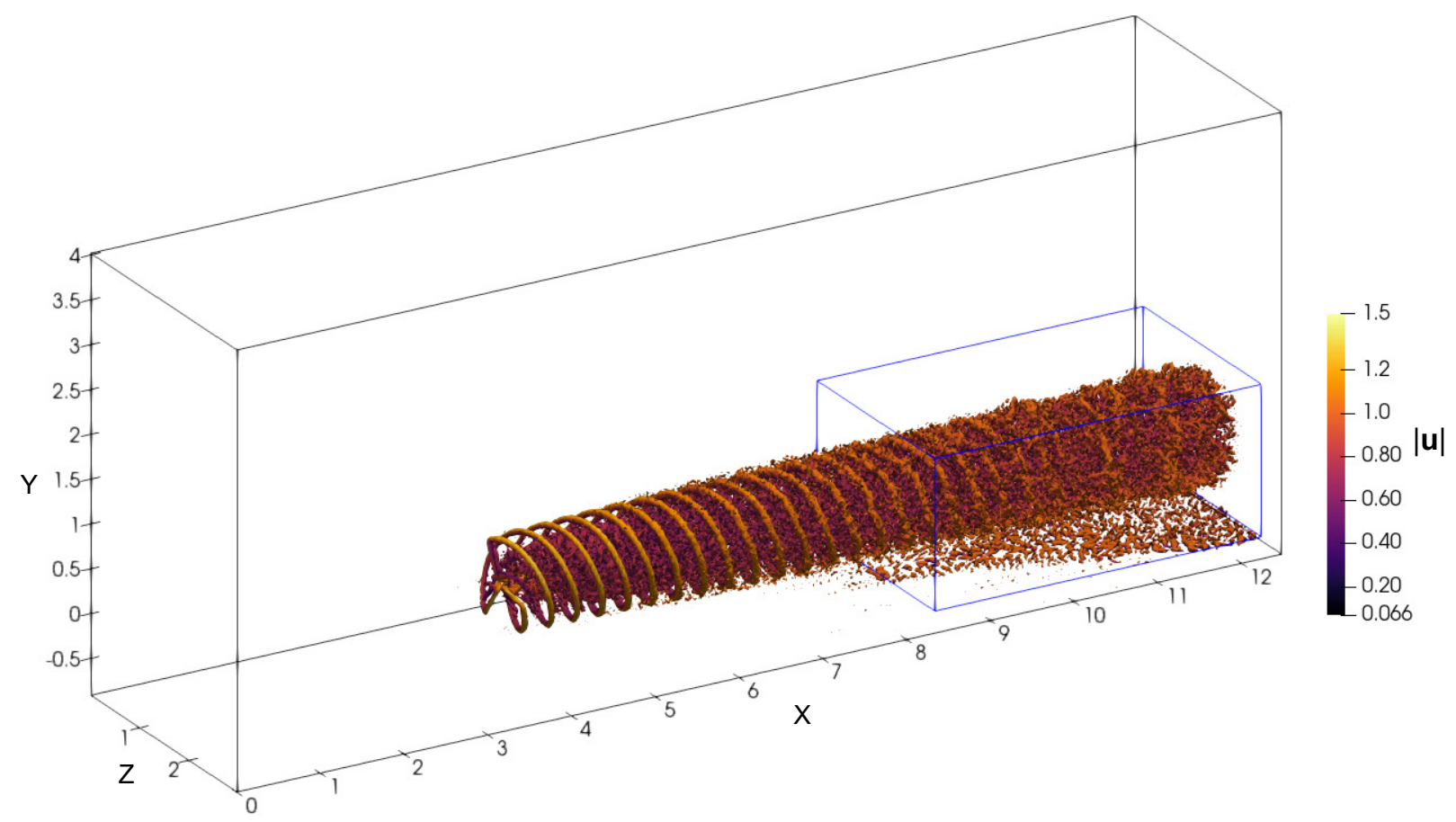

Figure 4: Snapshot of the flow field behind the wind turbine computed by LES: Q-criterion isosurfaces $(Q=0.5)$ coloured with the velocity magnitude (see legend). The box with blue edges represents the subdomain considered for the POD. 


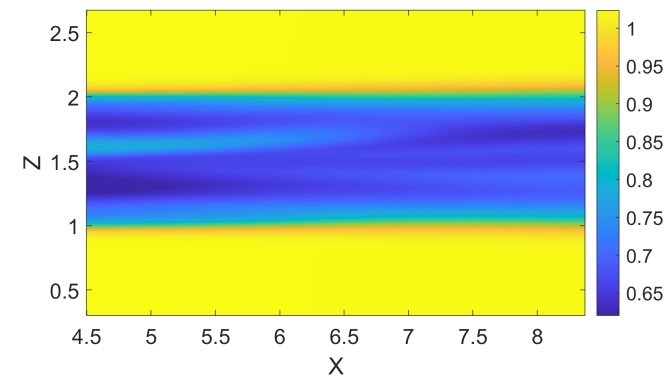

(a)

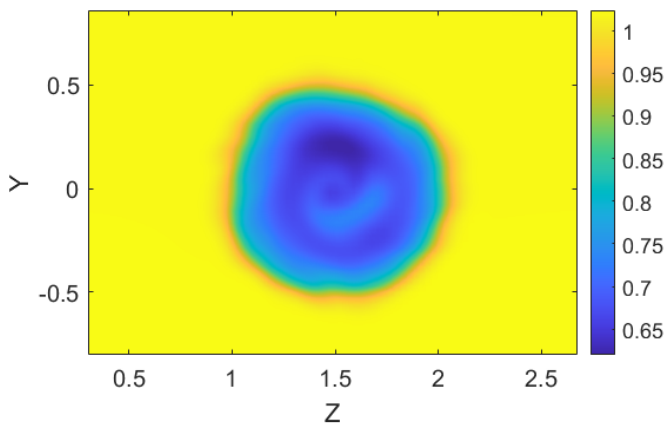

(c)

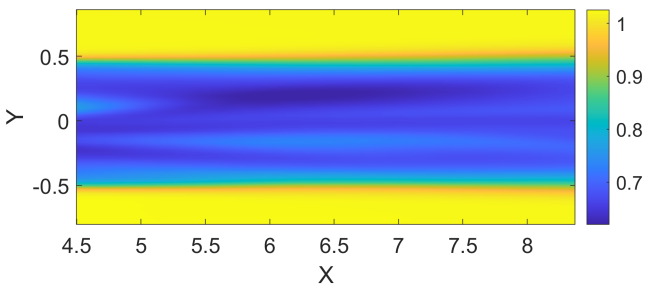

(b)

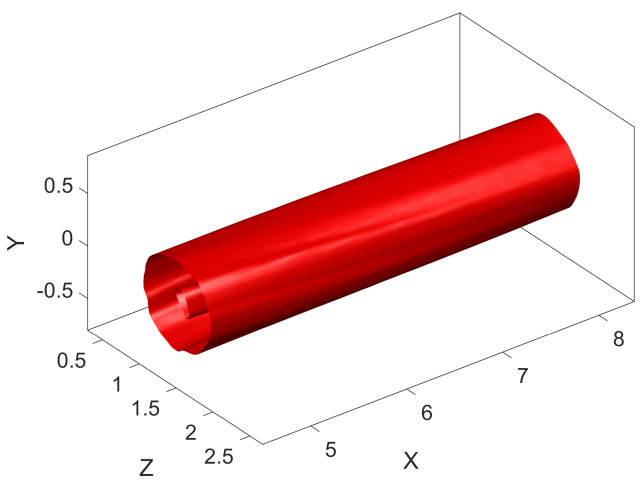

(d)

Figure 5: Streamwise velocity contours of the $0^{\text {th }}$ POD mode, in the $y=0$ (top left), $z=1.5$ (top right) and $x=6.5$ (bottom left) planes and three-dimensional plot of the isosurface $u=0.75$ (bottom right).

POD of the spatio-temporal flow fields behind the rotor has been carried out over a dataset made of $M=2575$ snapshots, after a thorough validation of the convergence of the POD modes with respect to the number of snapshots. Fig. 3 provides the convergence of the eigenvalues associated with the first 18 POD modes for different numbers of snapshots $1400 \leq M \leq 2575$. The first two modes converge very well already for the lowest value of $M$ considered, but the remaining ones need a larger number of snapshots to be well approximated.

The snapshots for the POD analysis are collected with a $\Delta t$ allowing for a 10 degrees rotation of the rotor. Thus, the dataset spans about 71 revolutions of the rotor blades. To allow a meaningful comparison with the stability analysis, the POD analysis has been carried out in a subdomain located in the far-wake, where the flow can be assumed to be approximately parallel. The inlet of the subdomain is set at a streamwise location for which the value of the coherent averaged kinetic energy drops below $3 \%$ of its maximum. According to this criterion, the resulting sub-domain extent, shown by the box in Fig. 4 is $\left[\begin{array}{ll}4.5 & 8.4\end{array}\right] \times[-0.80 .9] \times\left[\begin{array}{ll}0.3 & 2.7\end{array}\right]$, in the $x, y$ and $z$ directions, respectively. We have verified that the results of the analyses are robust with respect to the choice of the threshold for determining the far wake region. In this subdomain, the velocities have been down-sampled using a 1:5 ratio with respect to the computational grid. 


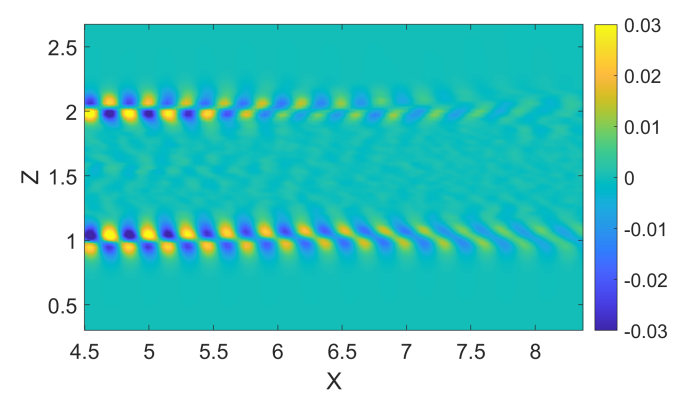

(a)

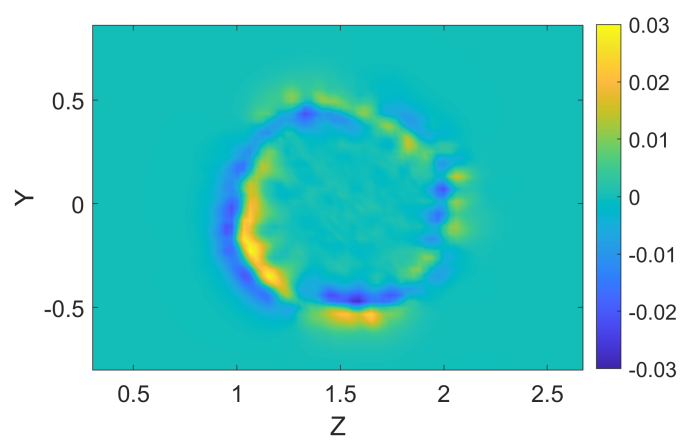

(c)

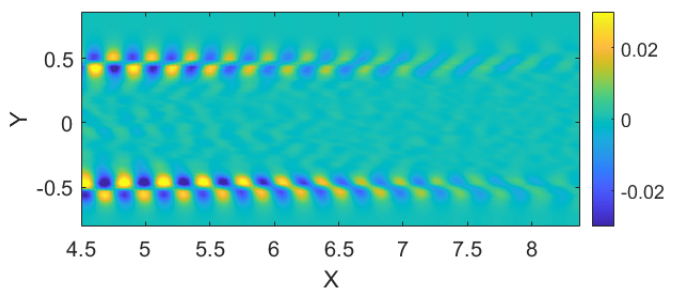

(b)

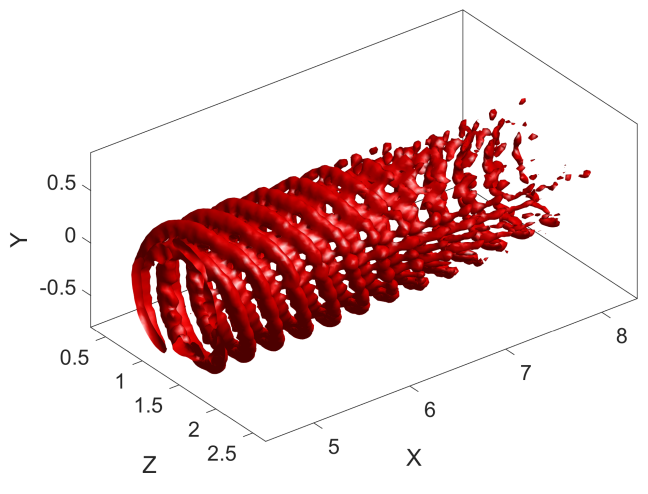

(d)

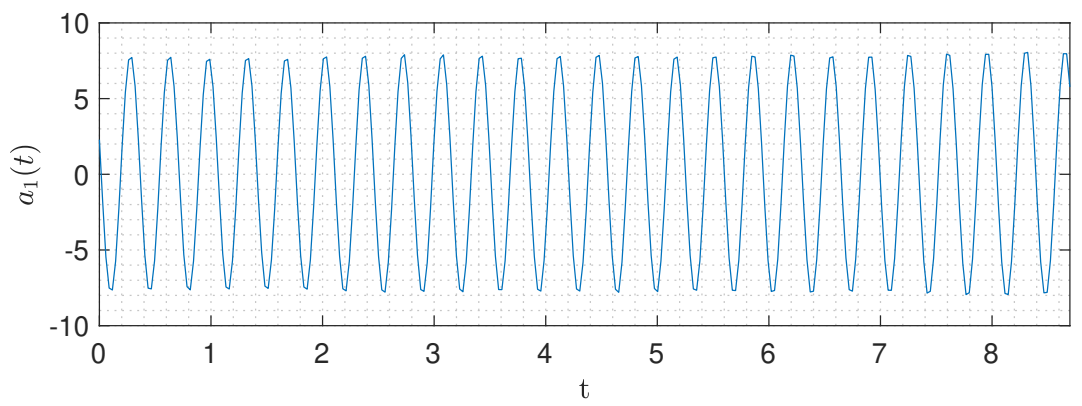

(e)

Figure 6: Streamwise velocity contours of the $1^{\text {st }}$ POD mode in the same planes of figure 5 (a-c) and in a three-dimensional visualization showing the isosurface of the streamwise velocity component of velocity $u=0.1 u_{\max }(\mathrm{d})$. The bottom plot represents the evolution of the associated temporal coefficient. 

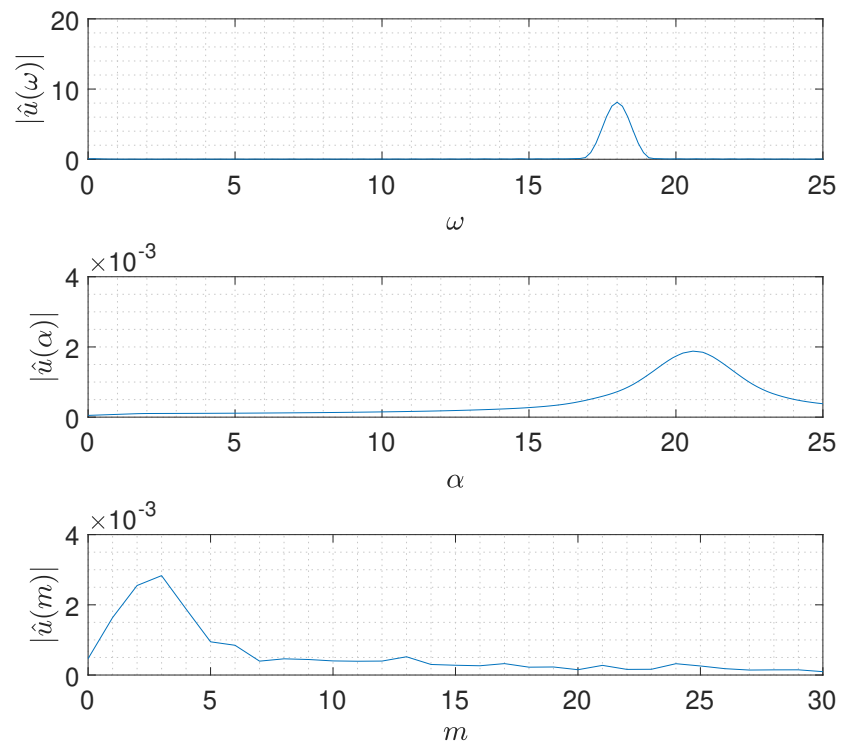

Figure 7: Spectra of the $1^{\text {st }}$ POD mode in time (top) and in the streamwise (middle) and azimuthal (bottom) directions.

\subsection{POD modes}

The POD method ranks the eigenvectors with respect to their energy content. The $0^{\text {th }}$ POD mode provides the time-averaged mean flow as shown in Fig. 5. The wake is slightly asymmetric in the vertical $(y, z)$ plane, and the near-wake flow rotates in the direction opposed to that of the rotor. The nacelle has been neglected, resulting in a nonphysical jet which develops at the center of the rotor for more than 3 diameters in the streamwise direction, and whose strength increases with the tip speed ratio [39]. However, in the far wake, the jet velocity gradient is smoothed out by the viscosity effect and the breakdown of the root vortex, as shown in the longitudinal planes provided in Fig. 5. The successive most energetic POD modes from 1 to 12 are paired: their associated eigenvalues are close to each-other and the time coefficients $a_{i}(t)$ have identical frequency spectra. The top frames of Fig. 6 provide the streamwise velocity contours of the $1^{\text {st }}$ POD mode in the $x-y$ and $z-y$ planes containing the rotor axis, showing a clear set of tip vortices fading out towards the end of the domain. The tip vortices are rather coherent in the region closest to the rotor, as shown by the streamwise velocity contours in the $x=6.5$ plane (Fig. $6 c$ ), but they begin to break down in the upper region of the wake towards the end of the computational sub-domain (Fig. 6d). The frequency content of the first POD mode is provided in Fig. 7, where the Fourier spectra in time and in the streamwise and azimuthal directions are shown, their wavenumbers being $\omega, \alpha$, and $m$, respectively. Since the mode is quasi periodic in time, as shown in Fig. 6 (e), the time spectrum has only one sharp peak at $\omega=18$, corresponding to three times the non-dimensional rotational frequency. The Fourier spectrum in the streamwise direction, resulting from the averaging of the Fourier spectra computed at different wall-normal and spanwise locations within the subdomain, shows a clear peak at $\alpha=20.92$, corresponding to the tip-vortices pitch. In the azimuthal direction, the Fourier spectrum, resulting from the averaging of different 

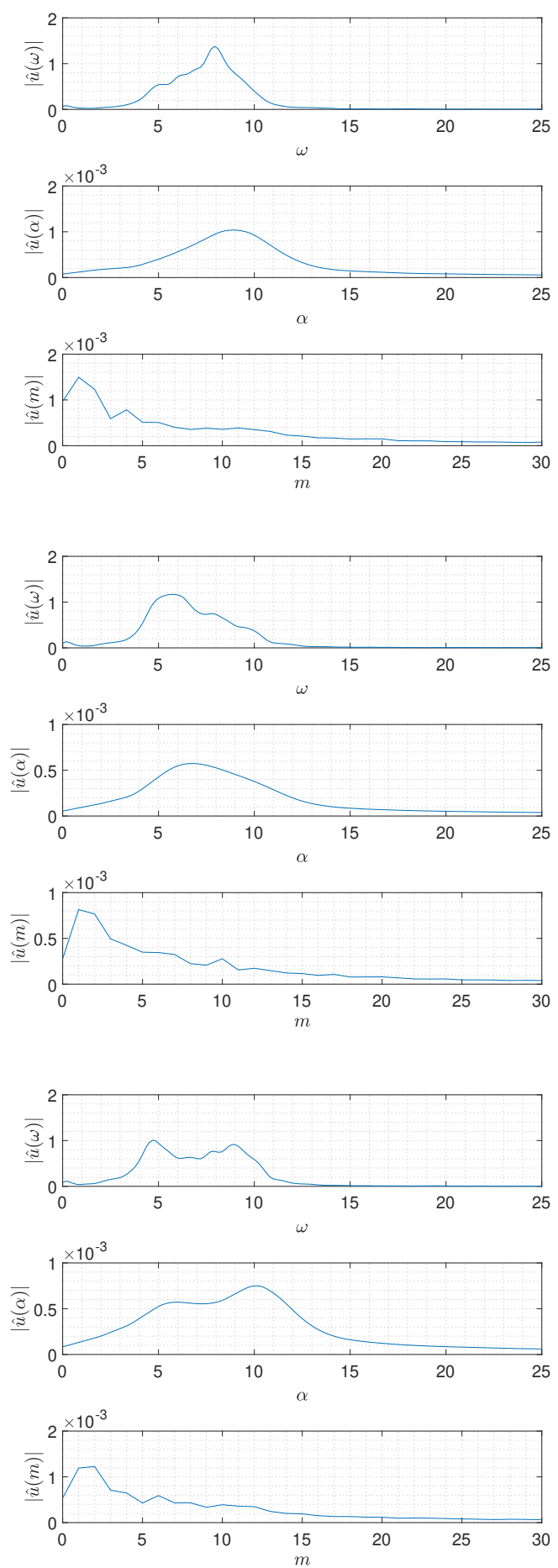
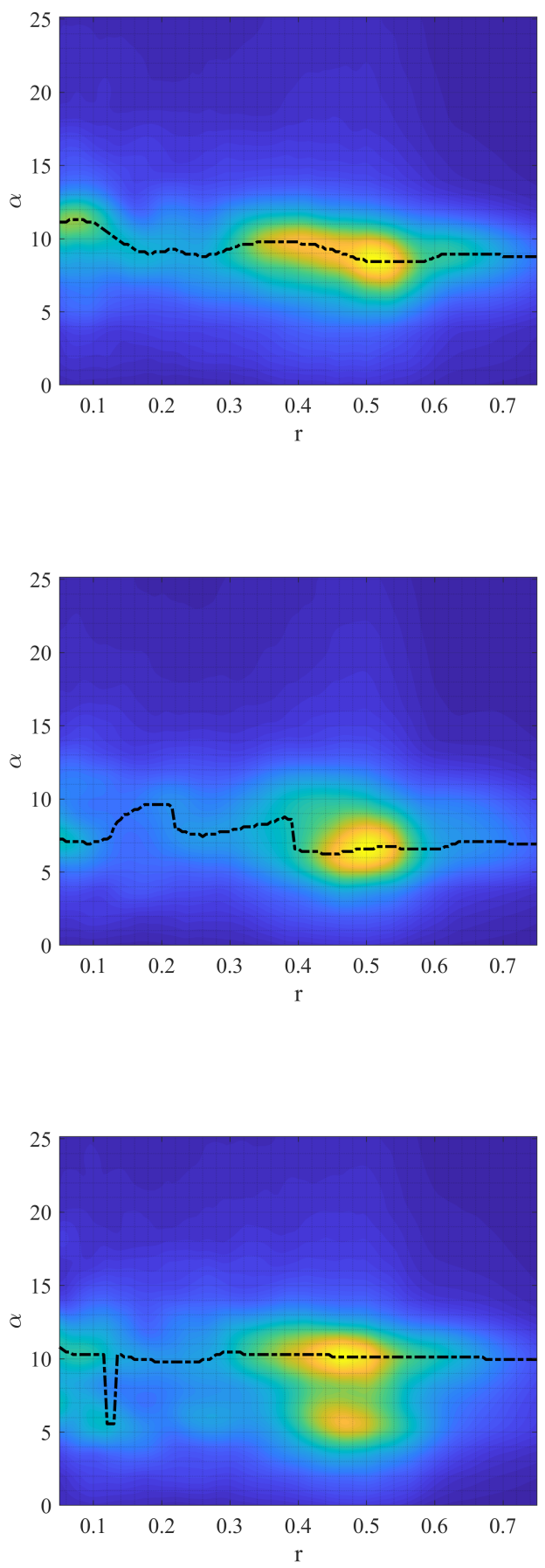

Figure 8: (Left) Fourier transform in time, streamwise and azimuthal directions of the $3^{\text {rd }}$ (top), $5^{\text {th }}$ (middle) and $7^{\text {th }}$ (bottom) POD modes. (Right) Streamwise frequency of the mode on the left for different radii, averaged in the azimuthal direction. The dashed-dotted line indicates the locus of the maximum amplitude of the Fourier modes for different values of $\alpha$ and radial position. 

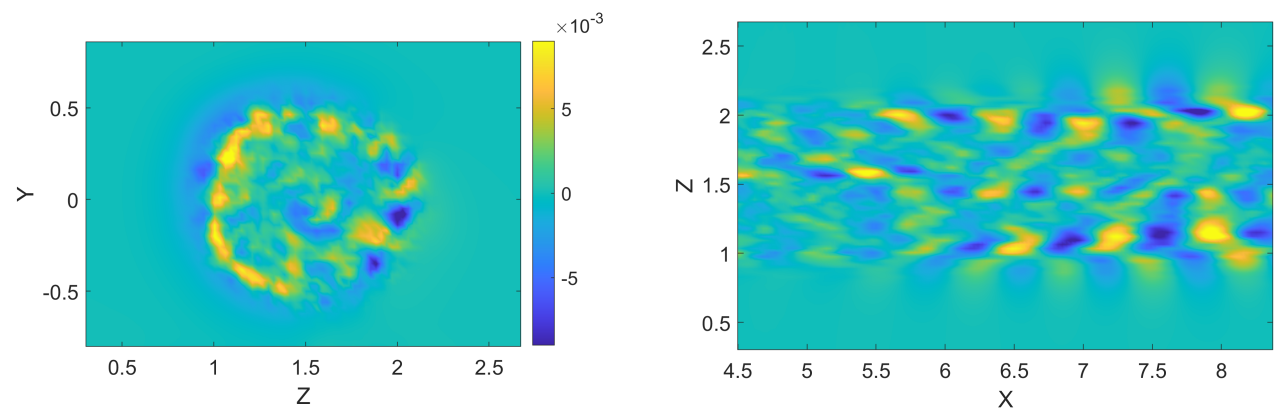

(a)
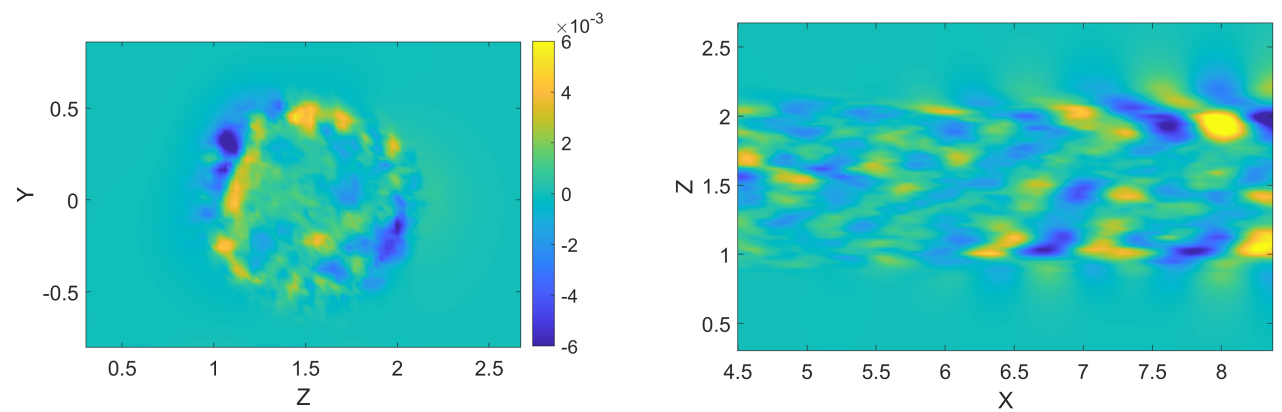

(b)
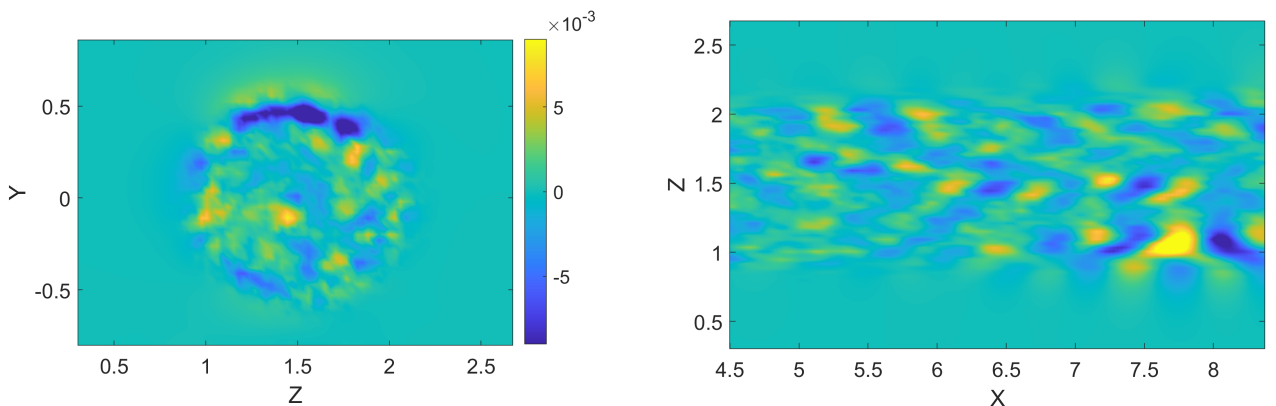

(c)

Figure 9: Streamwise velocity contours of the $3^{\text {rd }}$ (a) $5^{\text {th }}$ (b) and $7^{\text {th }}$ (c) POD modes in the $x=6.5$ (left) and $y=0$ (right) planes. 
spectra obtained for different radial and streamwise positions, peaks at the azimuthal wavenumber $m=3$, corresponding to the number of blades of the rotor. The successive pairs of modes have lower frequencies both in space and time, as shown by the spectra in Fig. 8 (left column) for the $3^{\text {rd }}, 5^{\text {th }}$ and $7^{\text {th }}$ POD modes (from top to bottom). Fig. 9 provides the streamwise velocity contours of the same POD modes (from top to bottom, respectively), showing that they are mostly located in the tip-vortices region, and to a lesser extent, in the core of the wake, where radial gradients due to the central jet are present. These modes capture the convective instabilities developing in the regions of high shear of the far wake.

One of these instabilities is the mutual inductance instability phenomenon investigated extensively by Sarmast et al. [40], which is characterized by a streamwise frequency about half the tip-vortex one. Here, the $3^{\text {rd }}$ POD mode has the main temporal and streamwise wavenumbers equal approximately to half the tip-vortex ones, namely $\omega \approx 8$ and $\alpha \approx 9$ (see the top left frame of Fig. 8). The radial distribution of the main streamwise wavenumbers of this POD mode, averaged on the azimuthal direction, provided in the top right frame of Fig. 8, shows that the main $\alpha$ peak is located at $r \approx 0.5$, corresponding to the tip vortices. A weaker peak is found also at $r \approx 0$, corresponding to the root-vortex system, associated to a slightly larger value of $\alpha$. Similarly to the mutual inductance instability discussed by Ivanell et al. [25], these POD modes are able to produce an outof-phase displacement of two consecutive tip vortices, leading to vortex pairing in the tip-vortex system, which promotes its break down.

The $5^{\text {th }}$ POD mode has a spatial structure similar to that of the $3^{\text {rd }}$ mode, but is characterized by slightly smaller temporal and streamwise wavenumbers, namely $\omega \approx 6$ and $\alpha \approx 7$. Moreover, the streamwise wavenumber appears to be only slightly dependent on the radial direction, being mostly localized in the outer part of the wake $(r \approx 0.5)$, as shown in Fig. 8. As it will be discussed in the following, this POD mode seems to be originated by a convective instability of the mean shear of the wake.

The $7^{\text {th }}$ mode presents a broader temporal and streamwise spectra, with two peaks at wavenumbers close to those of the previous modes. The bottom right frame of Fig. 8 shows that both peaks are mostly localized at the wake shear layer. In particular, the two main streamwise wavenumbers are $\alpha \approx 10.1$ and $\alpha \approx 6$, and two equally strong peaks are recovered in time, with $\omega \approx 4.7$ and $\omega \approx 8.9$. The spatial structure of this mode is very close to that of the previous ones, suggesting a similar physical mechanism at their origin. Finally, it is important to remark that the main azimuthal wavenumber of all these POD modes is $m=1$ (although the $7^{\text {th }}$ mode presents also a peak for $m=2$ ), confirming a strong similarity of the structure and physical origin of these modes.

In the next section, we consider these results as a benchmark for the linear stability analysis; in particular, the most amplified spatial wavenumbers $\alpha$ at each of the crosssectional planes are considered. The final goal is to verify to which extent the identified coherent structures are governed by linear mechanisms.

\section{Linear modeling of coherent structures}

We consider a linearization of the Navier-Stokes equations obtained by using a triple decomposition of the velocity field as proposed by Reynolds \& Hussain [38]

$$
\mathbf{u}(\mathbf{x}, t)=\overline{\mathbf{u}}+\widetilde{\mathbf{u}}+\mathbf{u}^{\prime} .
$$


The first term of the decomposition $\overline{\mathbf{u}}$ is the mean flow obtained by long-time averaging the flow field, whereas the remaining two terms describe the turbulent fluctuations; in particular, within the context of the triple decomposition, using a phase average, $\langle\cdot\rangle$, we make a distinction between organized waves containing all coherent time-periodic largescale motions, $\widetilde{\mathbf{u}}=\langle\mathbf{u}\rangle-\overline{\mathbf{u}}$ and the remaining incoherent turbulent fluctuations with zero phase average $\mathbf{u}^{\prime}$ (see $\S$ Appendix A). Here, we are interested in the dynamics of the organized wave $\widetilde{\mathbf{u}}$, governed by the equation

$$
\begin{gathered}
\frac{\partial \widetilde{\mathbf{u}}}{\partial t}=-\overline{\mathbf{u}} \cdot \nabla \widetilde{\mathbf{u}}-\widetilde{\mathbf{u}} \cdot \nabla \overline{\mathbf{u}}=-\nabla \widetilde{p}+\nabla \cdot\left[R e_{e f f}^{-1}\left(\nabla \widetilde{\mathbf{u}}+\nabla \widetilde{\mathbf{u}}^{T}\right)\right]+\mathbf{f}, \\
0=\boldsymbol{\nabla} \cdot \widetilde{\mathbf{u}},
\end{gathered}
$$

where $\mathbf{f}$ represents the nonlinear term, i.e., $\mathbf{f}=\boldsymbol{\nabla} \cdot \widetilde{\widetilde{\mathbf{u}}}$. Different assumptions on the latter term lead either to stability analysis $(\S 4.1)$ or optimal forcing analysis (§ 4.2). The frozen eddy-viscosity approach is used for modelling the Reynolds stresses. Such an approach is based on the effective Reynolds number $R e_{e f f}=\left(\frac{1}{R e}+\nu_{t}\right)^{-1}$, where the eddy-viscosity term, $\nu_{t}$, is a non-dimensional quantity dependent on the spatial coordinates. In this work, $\nu_{t}$ is computed as

$$
\bar{\nu}_{t}=-\frac{\overline{\mathbf{u}^{\prime} \mathbf{u}^{\prime}}: \overline{\mathbf{S}}}{2 \overline{\mathbf{S}}: \overline{\mathbf{S}}}
$$

\subsection{Two dimensional stability analysis}

Linearization of Eq.s (5) consists of neglecting the nonlinear term, $\mathbf{f}=\widetilde{\widetilde{\boldsymbol{u}}}$. Projection of the equations onto a divergence-free vector space provides

$$
\frac{\partial \mathbf{q}}{\partial t}=\mathbf{L q}
$$

L being the linearized phase-averaged Navier-Stokes operator projected onto the divergencefree vector space and $\mathbf{q}$ is the vector of the state variables. The asymptotic time evolution of an infinitesimal perturbation $\mathbf{q}$ of a given flow state is governed by the eigenspectrum of $\mathbf{L}$. Due to the very large dimensions of $\mathbf{L}$ after discretization of the linearized phaseaveraged Navier-Stokes equations, its leading eigenvalues cannot be easily obtained using direct eigenvalue computation. The eigenvalue problem is made computationally affordable by using the quasi-parallel hypothesis, for which the mean flow is supposed to be slowly varying in the streamwise direction. As shown in Fig. 11, providing the streamwise 


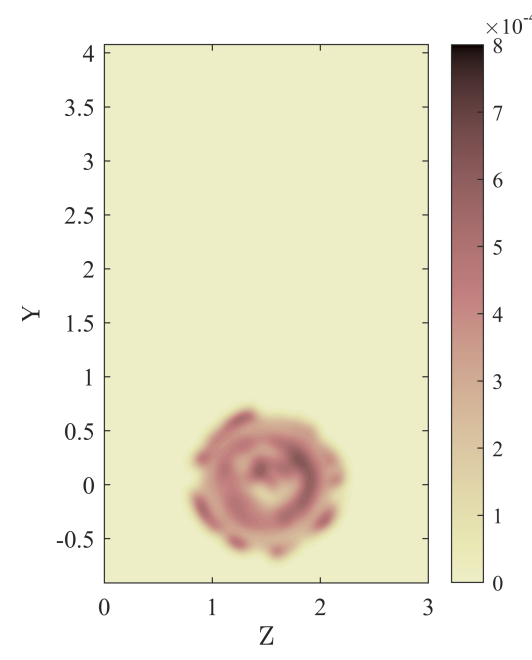

(a)

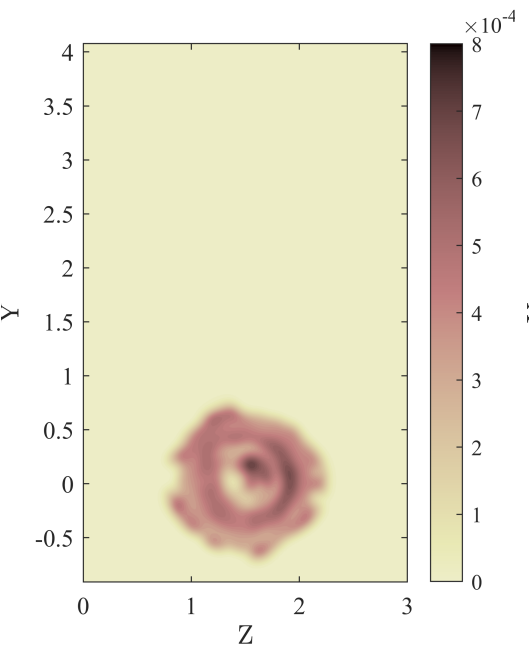

(b)

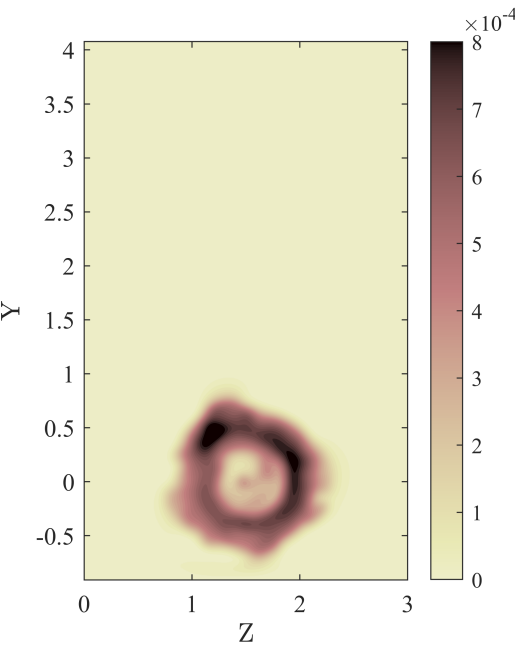

(c)

Figure 10: Turbulent viscosity $\nu_{t}$ at $x=3.5$ (a), $x=4.5(\mathrm{~b})$, and $x=6.5$ (c).

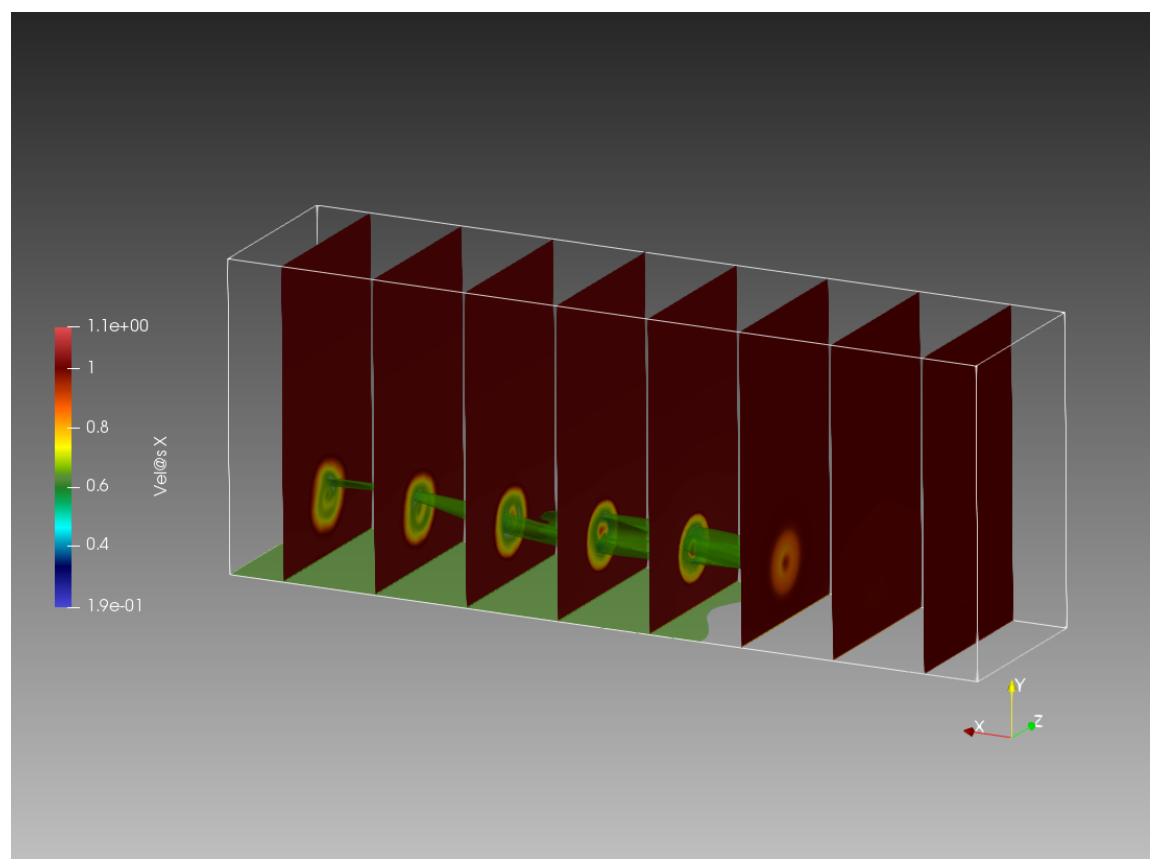

Figure 11: Time-averaged mean flow: iso-surfaces of the streamwise velocity component of the velocity and iso-contours of the same component at different cross-sections. 
velocity component of the mean flow velocity at different cross-sections, the mean flow is found to change slightly in the streamwise direction with respect to the other flow directions, making this hypothesis appropriate for the considered flow case. Following this rationale, we analyse the infinitesimal perturbations around the two-dimensional mean flow $\overline{\mathbf{u}}(y, z)$ introducing the ansatz

$$
\mathbf{q}(\mathbf{x}, t)=\hat{\mathbf{q}}(y, z) e^{i(\alpha x-\omega t)}+\text { c.c. },
$$

where $\hat{\mathbf{q}}$ is the Fourier-Laplace transform of $\mathbf{q}(\mathbf{x}, t)$, c.c. is its complex conjugate, $\omega$ is the complex temporal frequency, and $\alpha$ is the real wave number in the $x$ direction. Replacing the mean flow profiles and the ansatz (8) in the linearized system (7), the resulting problem is cast as an eigenvalues problem

$$
-i \omega \hat{\mathbf{q}}(y, z)=\mathbf{L} \hat{\mathbf{q}}(y, z)
$$

with eigenvectors $\hat{\mathbf{q}}_{k}(y, z)$ and eigenvalues $\omega=\omega_{r}+i \omega_{i}, \omega_{r}$ and $\omega_{i}$ being the growth rate and pulsation for each eigenvalue, respectively. Primitive-variable formulation is adopted, resulting after discretization in a linear operator $\mathbf{L}$ described by a $\left(N_{y} \times N_{z} \times 4\right) \times\left(N_{y} \times\right.$ $\left.N_{z} \times 4\right)$ matrix. The Chebyshev collocation method is employed in the wall-normal $(y)$ direction with a resolution of $N_{y}=75$, whereas a Fourier discretization is used in the spanwise $(z)$ direction with a resolution of $N_{z}=72$ collocation points. Dirichlet boundary conditions are imposed at upper and lower walls, and periodic boundary conditions are applied in the spanwise direction. The same discretization is adopted for the resolvent analysis detailed in the next section.

\subsection{Resolvent analysis}

While linear stability analysis identifies the flow structures and frequencies subject to exponential amplification, resolvent analysis establishes which kind of harmonic disturbances are amplified due to non-modal or quasi-resonance mechanisms, despite the system being asymptotically stable. We consider again the problem in Eq.s (5), where the term including the non-linearities $\mathbf{f}$ is modeled by a harmonic forcing and the following input-output linear problem arises

$$
\frac{\partial \mathbf{q}}{\partial t}=\mathbf{L q}+\mathbf{f}
$$

The approach we follow here is the one proposed in reference [38] and pursued in references $[18,23,46]$ among the others, where the turbulent Reynolds stresses related to the incoherent fluctuations $\mathbf{u}^{\prime}$ is included in the eddy viscosity model. An alternative approach consists of including in the external forcing also the instantaneous and averaged Reynolds-stress perturbations, as shown in references [30, 19, 32]. The real frequency $\omega \in \mathbb{R}$ is introduced in the ansatz $\mathbf{f}=\hat{\mathbf{f}} e^{i \omega t}$, under the assumption that the eigenvalues of $\mathbf{L}$ are confined to the stable half-plane; by Fourier transforming the state vector $\mathbf{q}$, we finally obtain the relation

$$
\hat{\mathbf{q}}=(i \omega \mathbf{I}-\mathbf{L})^{-1} \hat{\mathbf{f}}
$$

where $\mathbf{R}(\omega)=(i \omega \mathbf{I}-\mathbf{L})^{-1}$ is known as resolvent operator. Note that we consider a null initial condition, without loosing generality. In Eq. (11), the forcing $\hat{\mathbf{f}}$ is unknown, but it 
can be identified by maximizing the quotient

$$
R(\omega)=\max _{\hat{\mathbf{f}}} \frac{\left\|(i \omega \mathbf{I}-\mathbf{L})^{-1} \hat{\mathbf{f}}\right\|_{E}}{\|\hat{\mathbf{f}}\|_{E}} .
$$

The solution is associated to an optimization problem that can be solved by means of Singular Value Decomposition (SVD); the gain $R$ is obtained from the singular values, while the pair of modes are associated to the forcing term and the corresponding frequency response. Further details on the resolvent analysis are provided in $\S$ Appendix B.

\subsection{Stability modes}

In this section, we perform two-dimensional mean-flow stability analysis on different cross-sectional planes along the streamwise direction. Two considerations are in order. First of all, as a mean-flow analysis is considered, the comparisons that we carry out between the linear-model predictions and the turbulent flow generated by the wind turbine are mostly qualitative and needs to be assessed a posteriori. For this reason, we will consider the POD modes introduced in the previous section as a benchmark for the linear analysis. From a theoretical viewpoint, the analysis of spectral POD (SPOD) modes would be more appropriate $[29,37]$ : in fact, a single POD mode can contain multiple flow structures with different frequencies, originated from different physical mechanisms. Moreover, recent works have shown that a relation exists between the spectral POD modes and the frequency response obtained from a suitable decomposition of the resolvent operator [27, 48], under the assumption that the forcing harmonically drives the system at the different frequencies with equal spectral density (i.e. white noise). However, SPOD requires a larger number of snapshots to achieve convergence with respect to POD. Due to this limitation, we still adopt the POD and recover the frequencies information by means of Fourier transform during the post-processing for a quantitative analysis of its spectral content. A second aspect to be considered is the chosen ansatz, in which the streamwise wavenumber $\alpha$ is assigned; due to this hypothesis, the stability approach considered here can be regarded as local, as the two discretised coordinates are normal to the main direction of the flow [11].

Fig. 12 provides the stability eigenspectrum computed for the cross-sections $x=3.5$ and $x=6.5$, and different values of $\alpha$ ranging from $\alpha=3$ to $\alpha=10$. At $x=3.5$, the spectrum shows branches of unstable modes for each value of $\alpha$. A similar situation is obtained for the $x=6.5$ plane and $\alpha<8$, while stable modes are found for larger values of $\alpha$. The corresponding growth rate of the most unstable modes as a function of $\alpha$ are shown in Fig. 13(a) for $x=3.5, x=4.5$, and $x=6.5$. It appears that by moving downstream of the rotor, the maximum growth rate decreases and is achieved at values of $\alpha$ progressively smaller, going from $\alpha \approx 6$ at $x=3.5$ to $\alpha \approx 3.5$ at $x=6.5$. In Fig. $13(b)$, we can observe that the angular frequency of the most unstable modes is proportional to the value of $\alpha$ imposed for the stability analysis, with the relation $\omega_{r} \approx \alpha$ in all cases except at $x=6.5$ when a change can be observed at $\alpha \gtrsim 8.25$. We can conclude that stronger higher-frequency instabilities are predicted by the linear model in the vicinity of the rotor.

The eigenvectors corresponding to the most unstable eigenmodes at $x=3.5, x=4.5$ and $x=6.5$ are shown in Fig. 14a-c. In the two closest planes to the rotor, the most unstable modes are radially modulated as shown by the alternation of positive/negative 


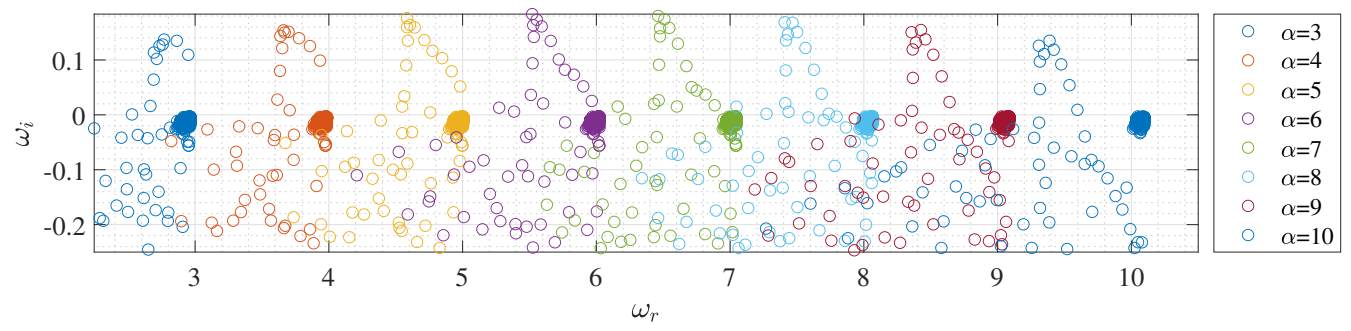

(a)

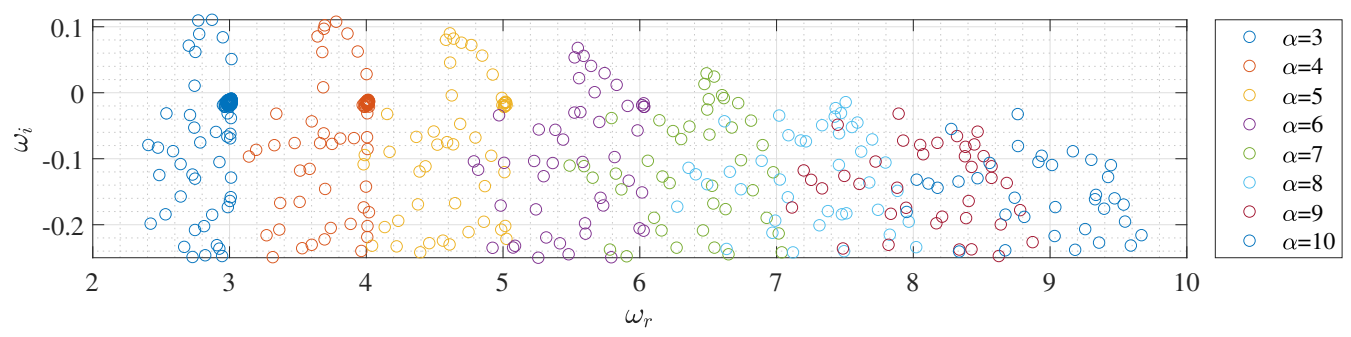

(b)

Figure 12: Eigenvalue spectra at $x=3.5$ (a) and $x=6.5$ (b) for different values of $\alpha$ reported in the legend.

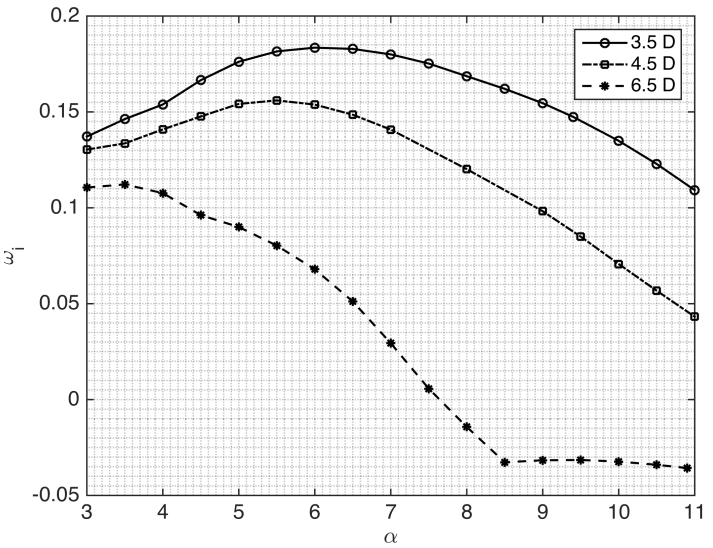

(a)

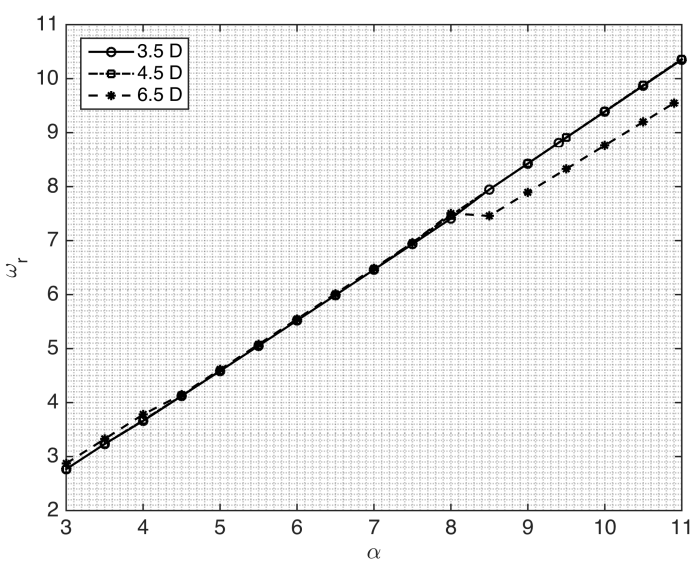

(b)

Figure 13: (a) Growth rates of the most unstable eigenmodes for different streamwise frequencies $\alpha$. (b) Temporal frequencies associated to the growth rates in (a). 

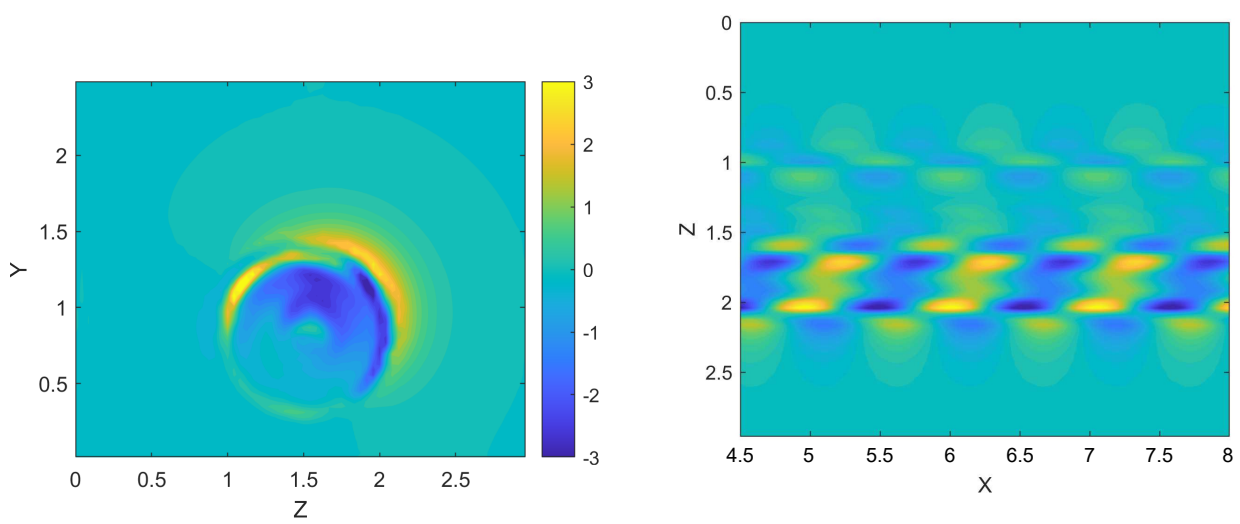

(a)
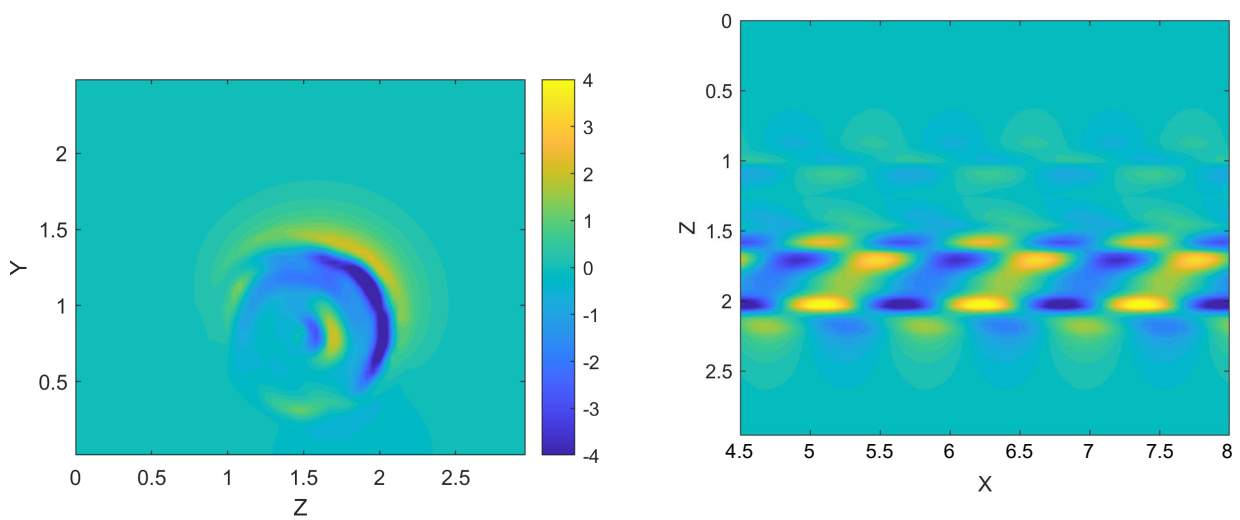

(b)
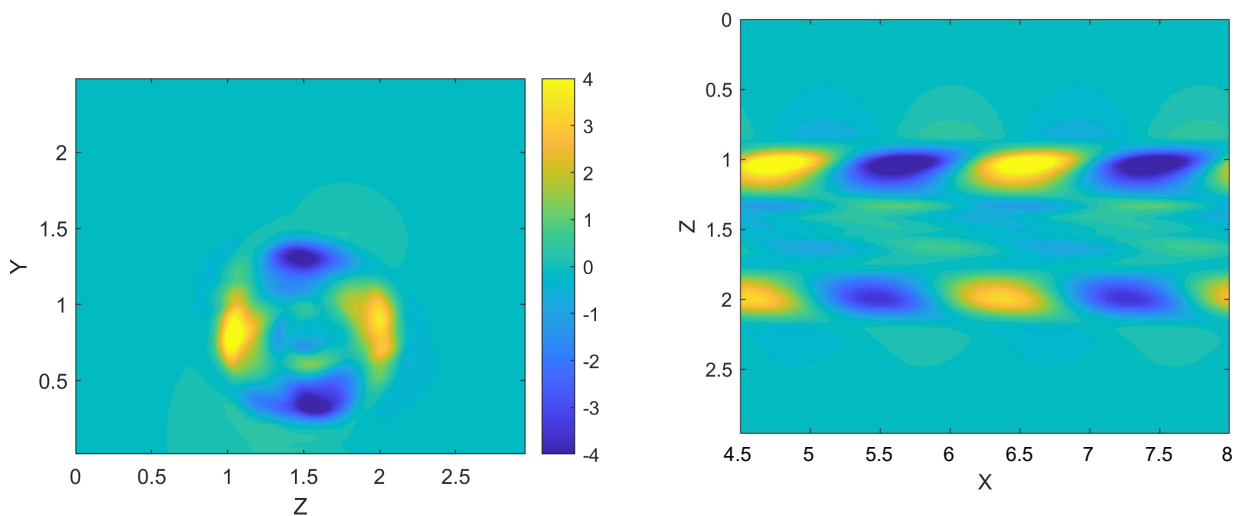

(c)

Figure 14: Streamwise velocity component of the most unstable eigenmode for $\mathrm{x}=3.5$ and $\alpha=6$ (a), $\mathrm{x}=5.5$ and $\alpha=5.5(\mathrm{~b})$, and $\mathrm{x}=6.5$ and $\alpha=3.5$ (c) in a $z-y$ (Left) and $x-z$ (Right) plane, after reconstruction of the perturbation in the streamwise direction. 

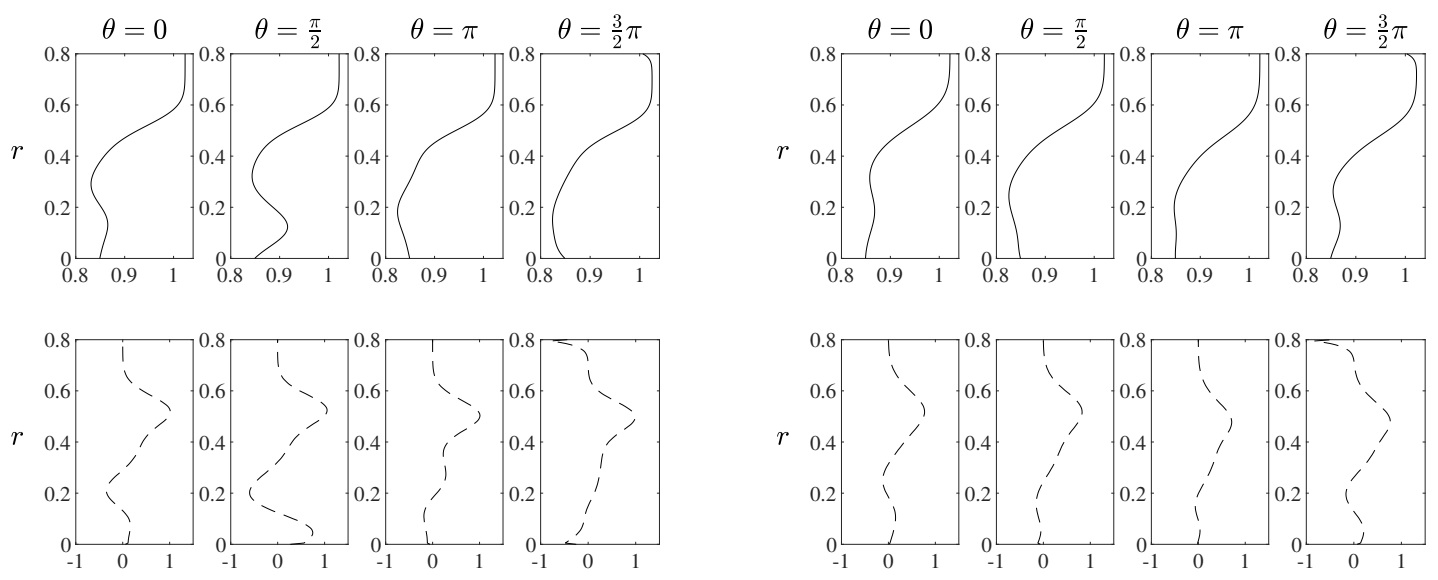

(a) $\bar{u}(-), \frac{\partial \bar{u}}{\partial r}(----)$ at $x=3.5$

(b) $\bar{u}(-), \frac{\partial \bar{u}}{\partial r}(----)$ at $x=6.5$

Figure 15: Mean-flow streamwise velocity profiles

) and corresponding radial derivatives (----) at four azimuthal positions, $\theta=0, \pi / 2, \pi, 3 \pi / 2$, corresponding to the right, bottom, left and top part of the wake, respectively, in the cross-flow planes at $x=3.5$ (a) $x=6.5$ (b).

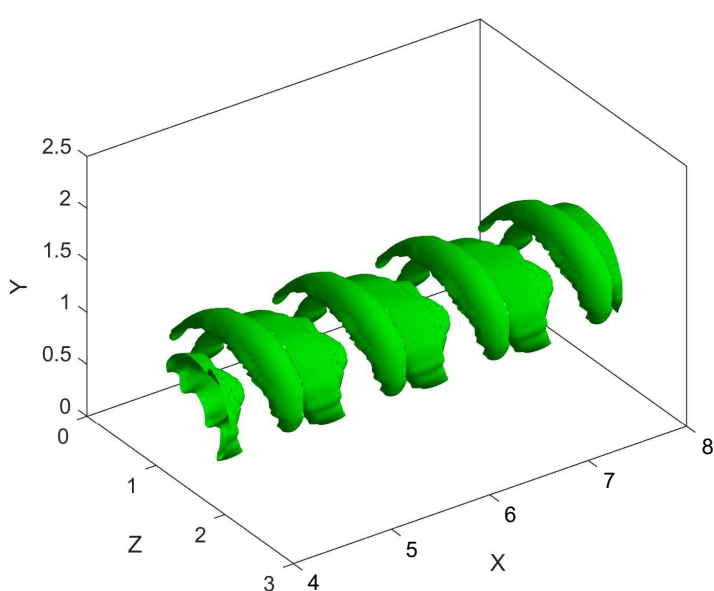

(a)

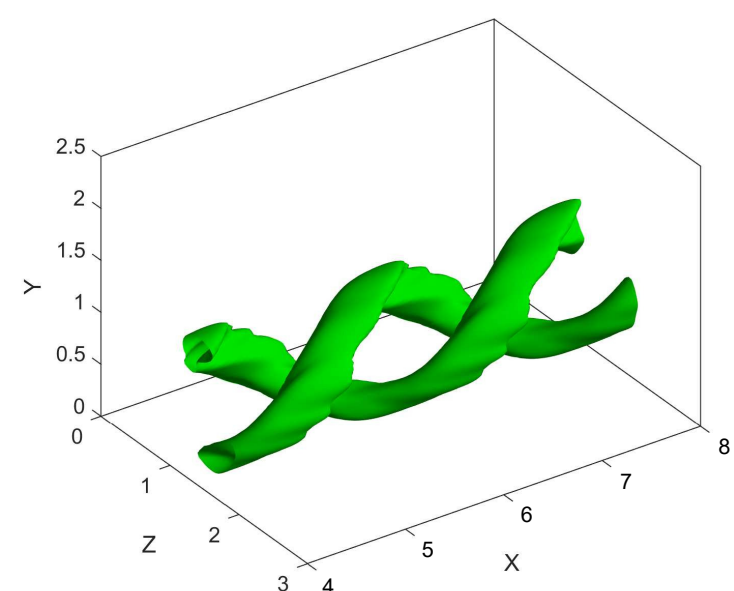

(b)

Figure 16: Isosurface of streamwise velocity of the three-dimensional reconstruction of the most unstable mode for (a) $x=3.5, \alpha=6$, and (b) $x=6.5, \alpha=3.5$.

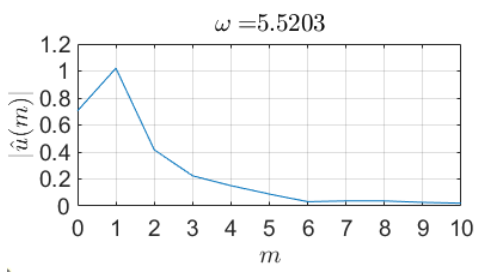

(a)

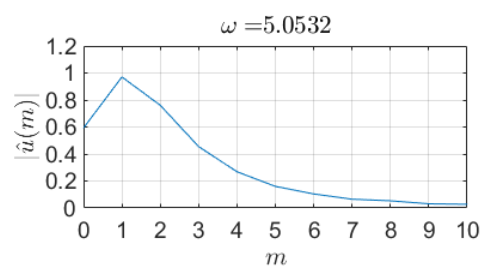

(b)

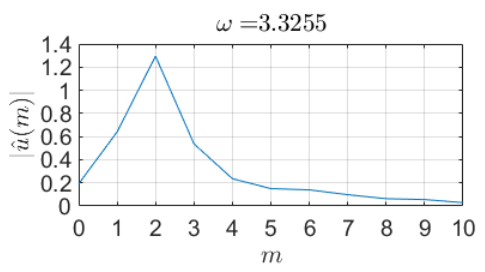

(c)

Figure 17: Fourier transform in the azimuthal direction of the streamwise velocity component of the eigenmode at $\mathrm{x}=3.5$ and $\alpha=6(\mathrm{a}), \mathrm{x}=5.5$ and $\alpha=5.5(\mathrm{~b})$, and $\mathrm{x}=6.5$ and $\alpha=3.5$ (c), all representing the maximum growth rate in the considered plane. 
streamwise disturbances, mostly localized at $r \approx 0.5$ and along the centerline $r \approx 0$. A streamwise view of the $u$ component of these modes is provided in the right frames of Fig. 14.

Focusing on the most unstable modes at $x=3.5$ and $x=4.5$, we observe that their structure is mostly localized on the right region (with respect of an observer looking downstream). This asymmetry is attributed to the mean flow, characterized by different shear intensities in the azimuthal direction, as shown in Fig. 15 for four different azimuthal angles $\theta=\{0, \pi / 2, \pi, 3 \pi / 2\}$ (varying counter-clockwise starting from the plane with $y=0$ and positive $z$, corresponding to $\theta=0$ ) at $x=3.5$ and $x=6.5$. In particular, Fig. 15 shows that, in the plane closest to the rotor, the mean flow shear is less pronounced in the left part of the wake, whereas, in the right region of the wake, the streamwise velocity profile presents two inflection points in the radial direction, i.e. $\partial^{2} \bar{u} / \partial r^{2}=0$. At $x=6.5$, despite an inflection point can be found as well, the radial shear is weaker and more uniform in both radial and azimuthal directions, probably due to the mean kinetic energy entrainment. Thus, the unstable mode has almost the same intensity at different azimuthal positions. All these features can be summarised by analysing the three-dimensional rendering of the modes in Fig. $16 a-b$, for $x=3.5, \alpha=6$ and $x=6.5$, $\alpha=3.5$, respectively; here, the most unstable mode at $x=3.5$ shows streamwisealternating and azimuthally-elongated patches following the mean-flow shear in the upperright part of the wake, while the mode at $x=6.5$ develops a double-helix structure.

We consider now the POD modes analysed in Section 3.1. The first pair of modes, which capture the tip-vortex helices, is not considered. In fact, the tip vortices are "forced" by the rotor and do not emerge as an instability of the wake mean flow. By analysing the spectral content of the successive POD modes, we observe that the main streamwise angular frequency found in the $5^{\text {th }}$ POD mode and in the second peak of the $7^{\text {th }}$ POD mode (see the bottom frame of Fig. 8), $\alpha \approx 6$, is close to the streamwise frequencies of the most unstable modes found at $x=3.5$ and $x=4.5$, namely $\alpha=6$ and $\alpha=5.5$, respectively. The spatial structure of the $u$ component of these two unstable modes is provided in the two upper right frames of Fig. 14, where it is possible to observe that the overall structure resembles the POD modes $3-7$. Moreover, a Fourier transform in the azimuthal direction, provided in Fig. 17, shows that the main azimuthal wavenumber of the most unstable mode found in the plane closest to the rotor coincides with that of the most energetic POD modes, namely, $m=1$. Whereas, the most unstable mode for $x=6.5$, found for $\alpha=3.5$, is characterized by main azimuthal wavenumber $m=2$, and by structures of longer wavelength in the streamwise direction. Neither this wavenumber, nor the double-helix structure of this eigenmode, shown in Fig. 16, is recovered in the main POD modes. This is probably due to an insufficient length of the computational domain in the streamwise direction. In fact, the angular frequency $\alpha \approx 3.5$ corresponds to a wavelength $\lambda_{x}=\frac{2 \pi}{\alpha} \approx 1.8$. Since the unstable mode with $\alpha \approx 3.5$ emerges at $x=6.5$, only one wavelength can be contained in this portion of the domain, which may be insufficient to capture unambiguously such an instability.

\subsection{Optimal forcing analysis}

In the previous section, we noticed that the most unstable mode found at $x=6.5$ is not recovered by the POD. However, at the same location, the mean flow is linearly stable for $\alpha>7.5$. This allows us to complement the linear stability analysis with a resolvent 


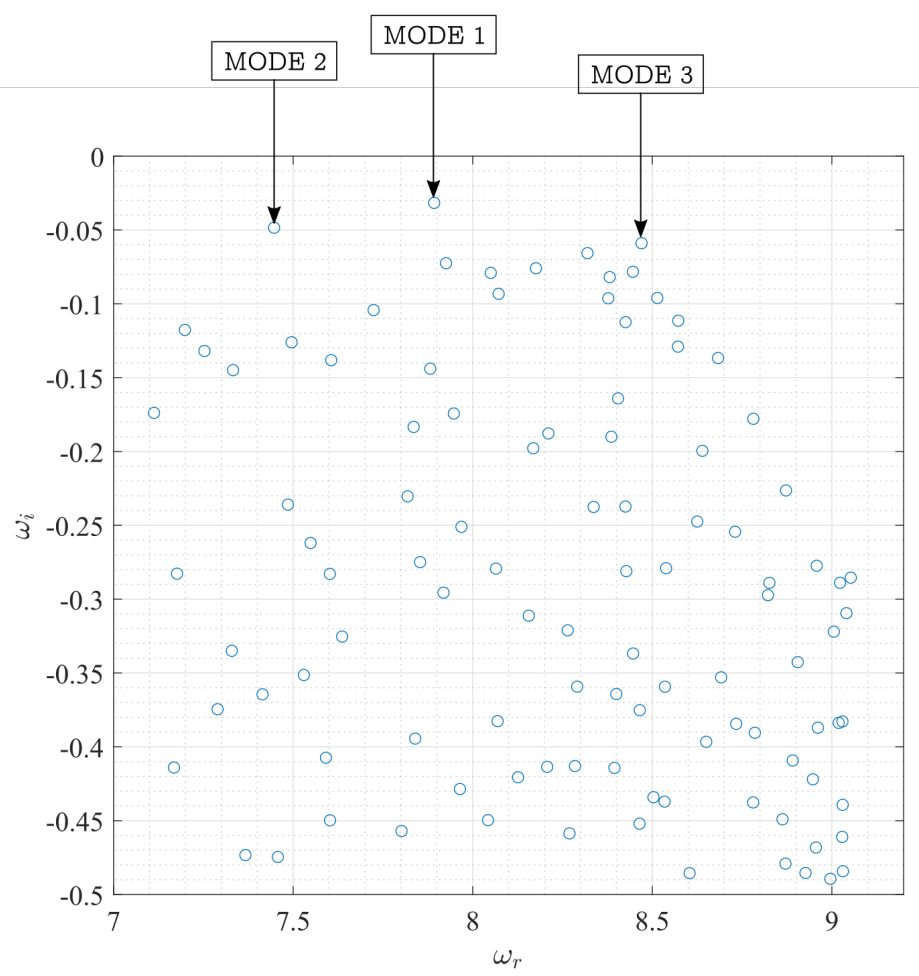

Figure 18: Eigenvalue spectrum for $x=6.5$ and $\alpha=9$.

analysis, which enables to scrutinize the response of the system when harmonically driven by real frequencies and to identify the corresponding optimal forcing. In order to do so, we consider the main streamwise frequency that characterize the third POD mode, namely $\alpha=9$ (see the top frames of Fig. 8). Inspecting the corresponding eigenspectrum provided in Fig. 18, we notice that there are three modes lying very close to the neutral axis, whose spatial structures are shown in Fig. 19. The two of them characterized by a (slightly) lower angular frequencies are mostly localized in the region $r \approx 0$, showing positive/negative streamwise disturbances alternating in the azimuthal direction. Whereas, the mode with highest temporal wavenumber is mostly localized in the $r \approx 0.5$ region, showing a more complex flow structure. This correspondence between localization and wavenumber is consistent with what has been observed in the $3^{\text {rd }}$ POD mode, being characterized by slightly larger wavenumbers in the root region with respect to the tip one (see the top right frame of Fig. 8). Moreover, Fourier transform in the azimuthal direction shows that all of these modes are characterized by main azimuthal wavenumber $m=1$ (see right frame of Fig. 19). The azimuthal Fourier spectrum of mode 3 (see bottom right frame of Fig. 19) has a second peak with equivalent amplitude at $m=2$ and a weaker peak at $m=4$. As already noticed, the POD mode taken here as reference comprises different structures with a different localization and frequency. Therefore we attempted to reconstruct the POD mode by combining the three least stable modes mentioned above with coefficients $c_{1}=0.25, c_{2}=0.15, c_{3}=0.6$ which have been chosen after some trials followed by comparison with the POD mode. Fig. 20 provides the streamwise velocity contours of this combination, which shows a strong resemblance with the overall structure of the third POD mode given in Fig. 9. 

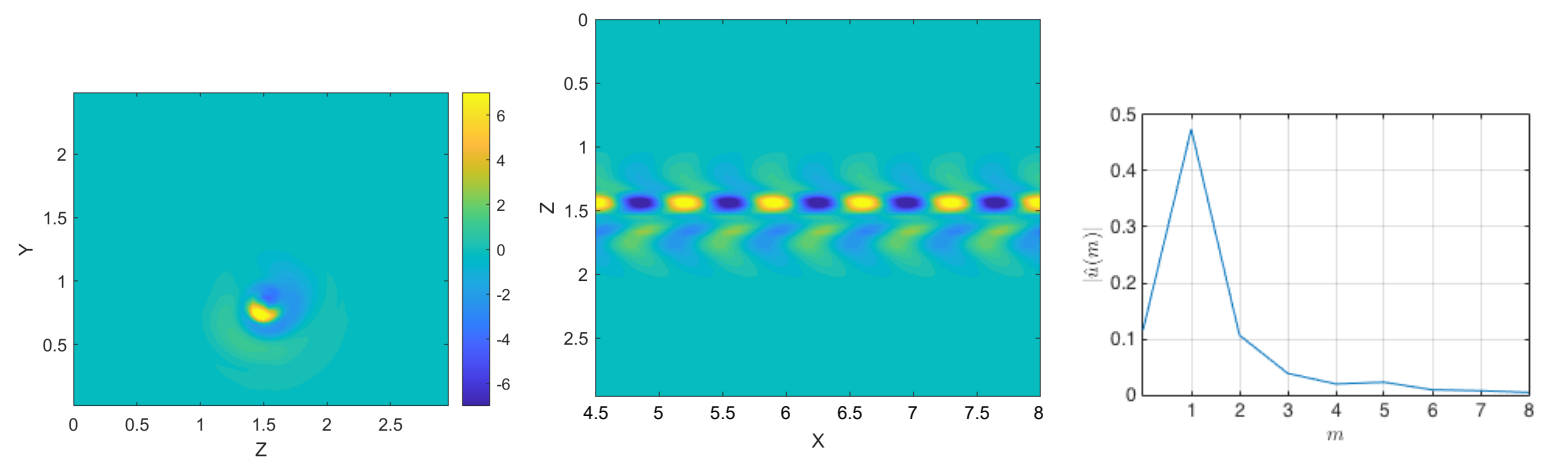

(a)
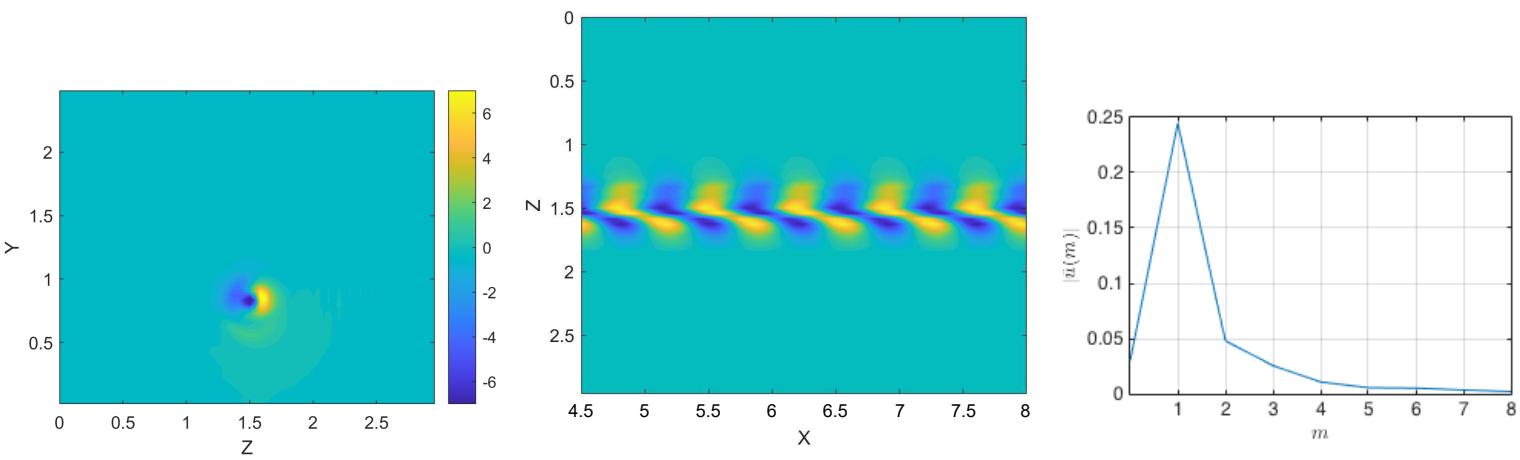

(b)
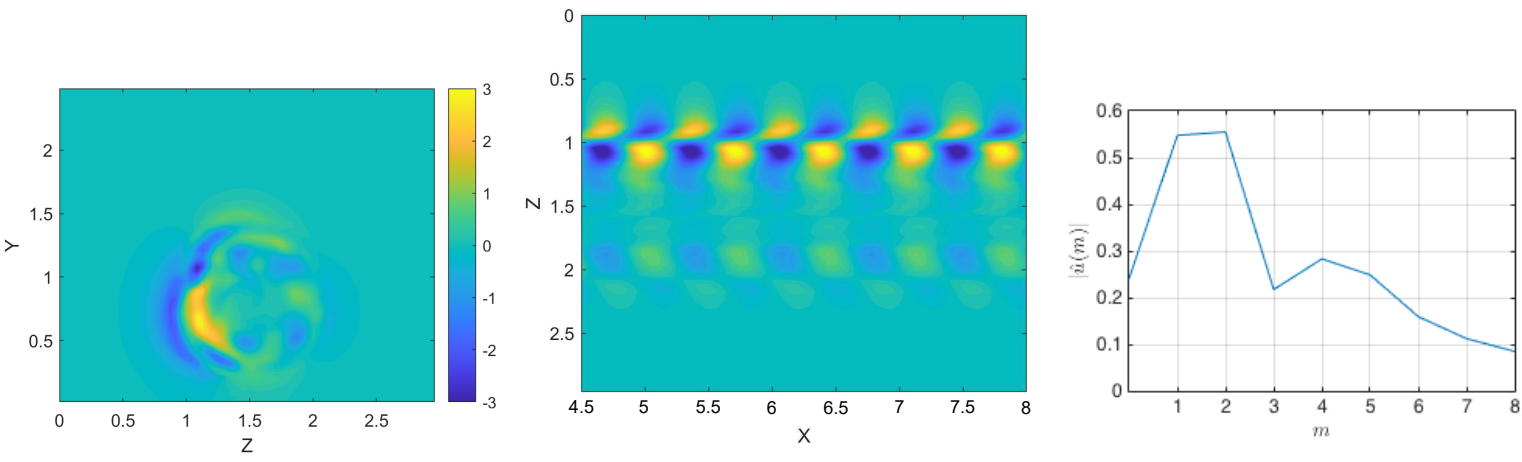

(c)

Figure 19: First (a), second (b) and third (c) most unstable modes obtained for $\mathrm{x}=6.5$ and $\alpha=9$ : streamwise disturbance in the $z-y$ (left) and in the $x-z$ plane (middle) and associated Fourier amplitudes in the azimuthal direction (right). 

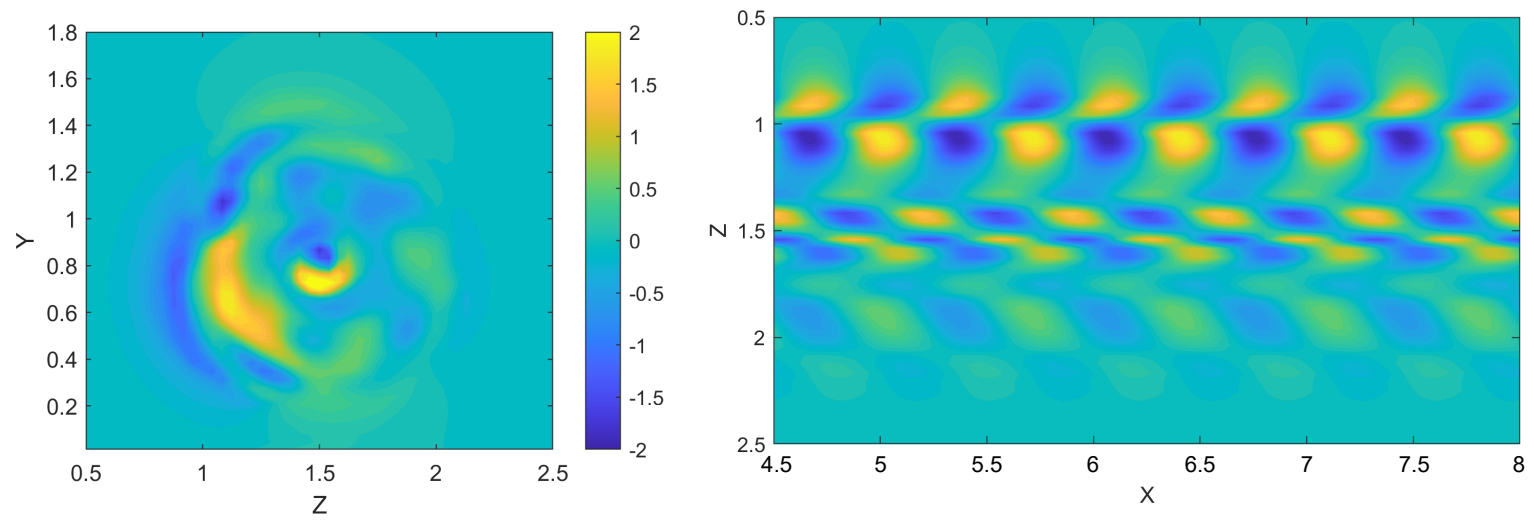

Figure 20: Linear superposition of the three main eigenmodes of the energy spectrum at $x=6.5$ and $\alpha=9$ : streamwise disturbance in the $z-y$ (left) and in the $x-z$ plane (right)

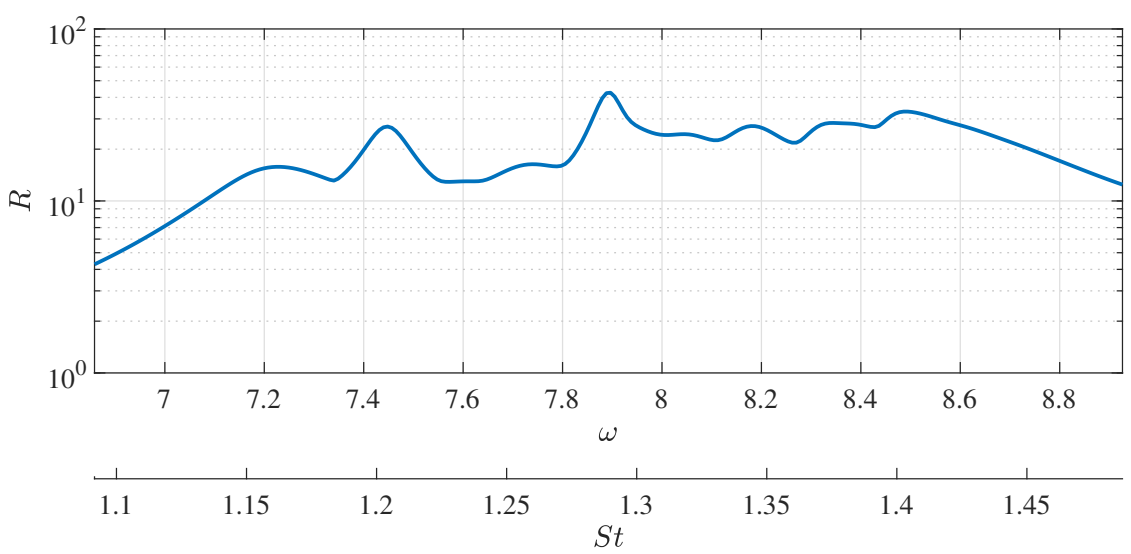

(a) $x=6.5$

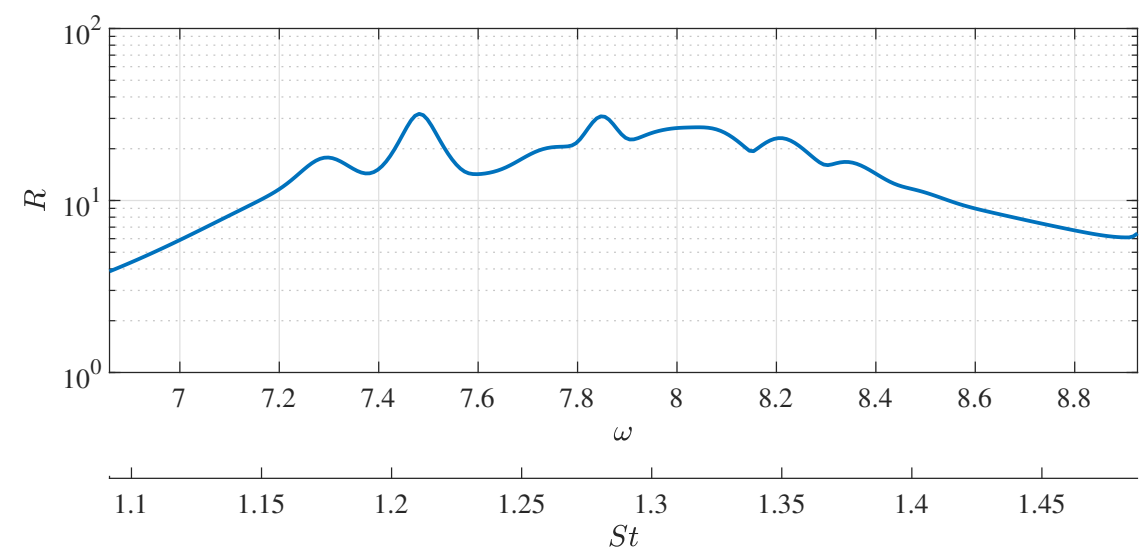

(b) $x=7.5$

Figure 21: Resolvent norm at different cross-sections for $\alpha=9$, versus the frequency $\omega$ and the Strouhal number $S t$. 

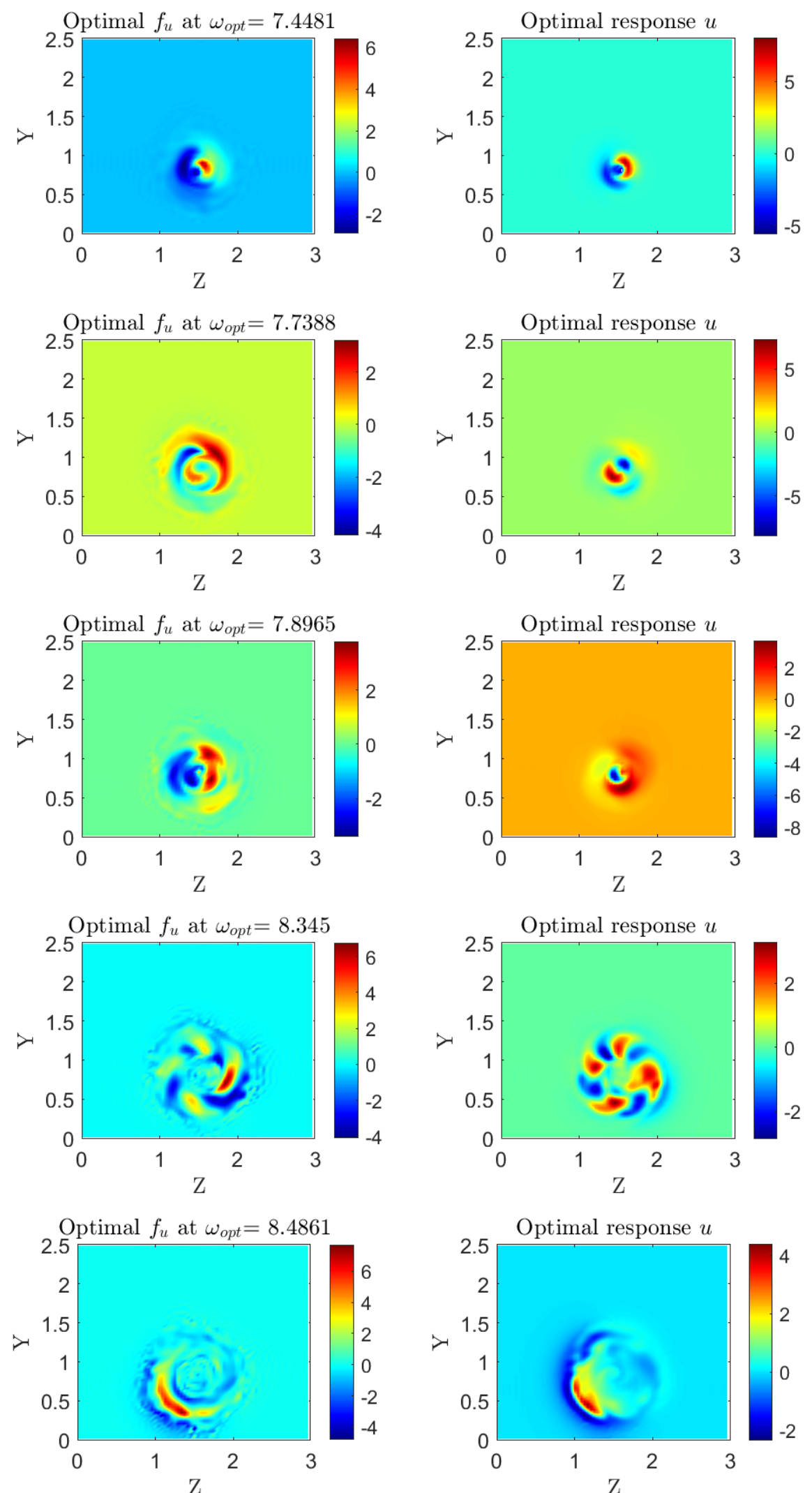

Figure 22: Streamwise velocity component of the optimal forcing (left) and responses (right) for different frequencies $\omega_{\text {opt }}$ for $x=6.5$ and $\alpha=9$. 


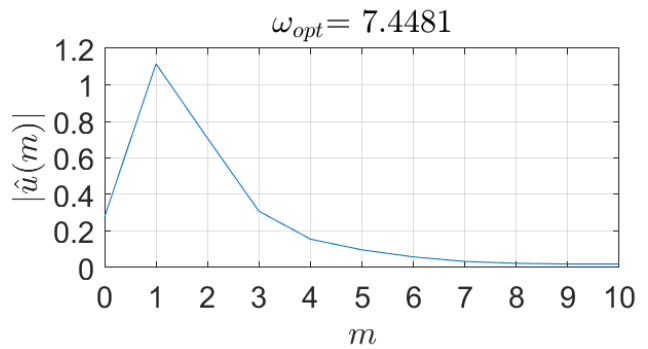

(a)

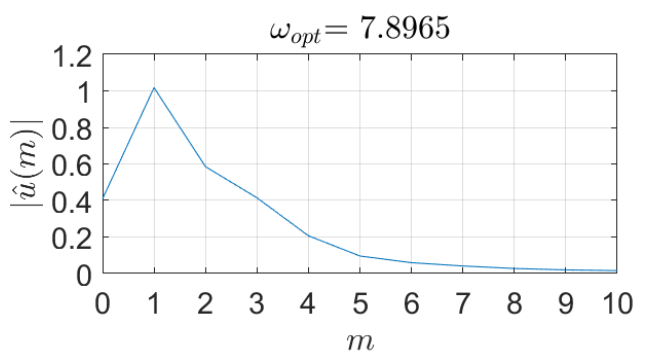

(c)

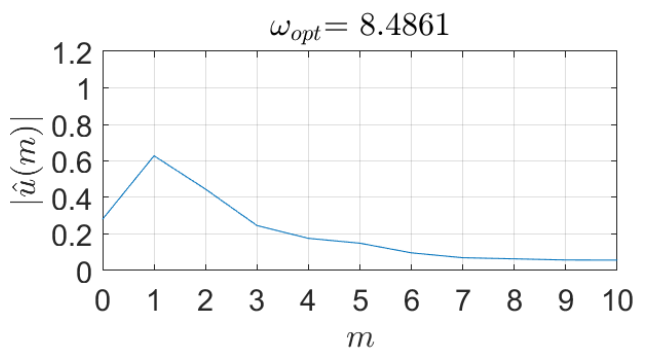

(e)

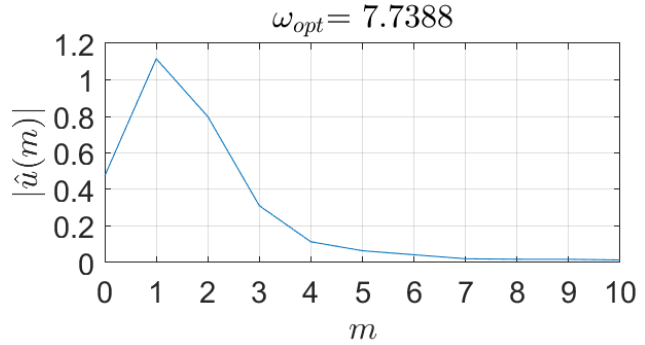

(b)

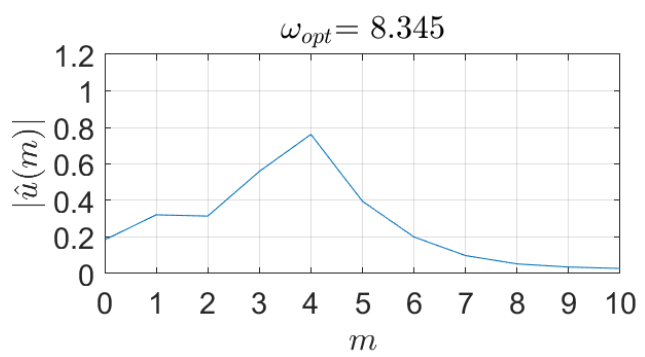

(d)

Figure 23: Fourier transform in the azimuthal direction of the optimal responses at different frequencies $\omega_{\text {opt }}$ indicated within the plots for $x=6.5$ and $\alpha=9$. 

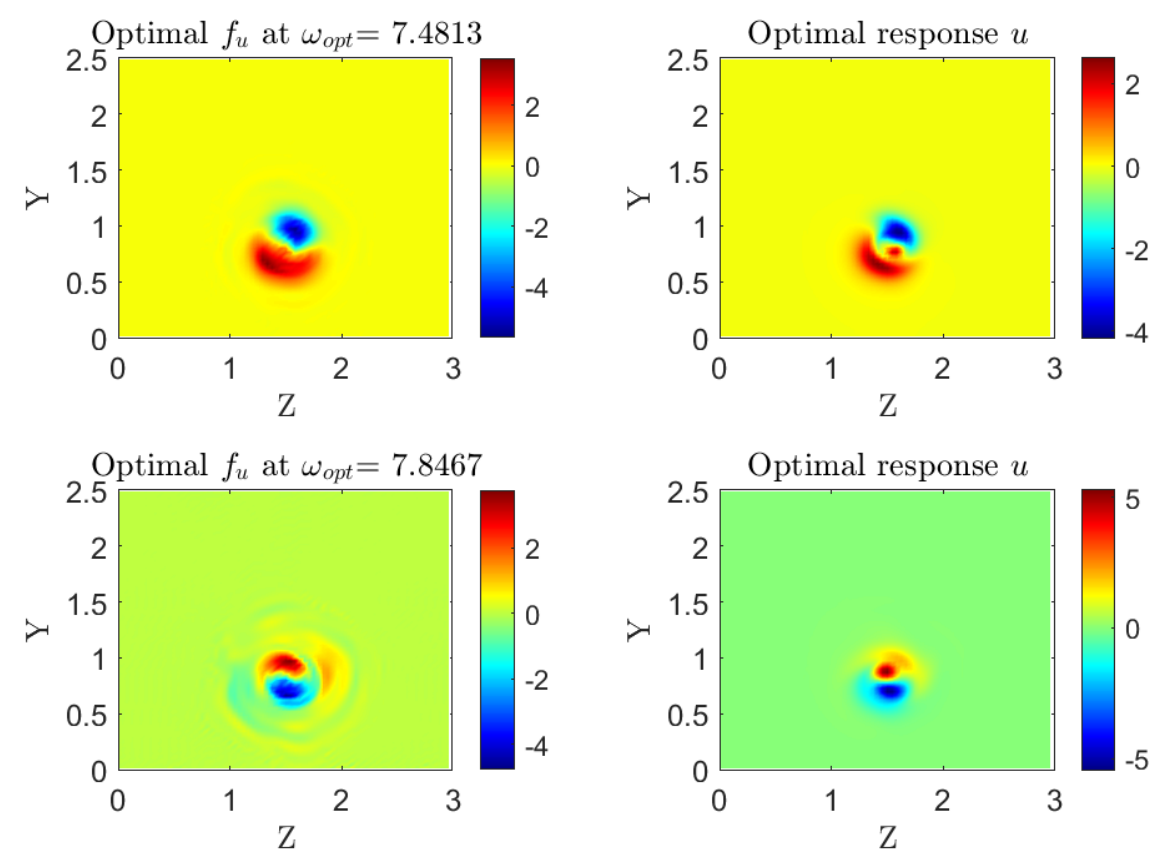

Figure 24: Streamwise velocity component of the optimal forcing (left) and responses (right) for different frequencies $\omega_{\text {opt }}$ for $x=7.5$ and $\alpha=9$

Fig. 21 shows the resolvent norm $R(\omega)$, providing the maximum energy gain for a given forcing in the range $6.8<\omega<9$, corresponding to a Strouhal number $1.1<S t<1.5$. One can notice that the main three peaks correspond to the frequencies of the least stable modes of the eigenspectrum, suggesting a quasi-resonance mechanism at these particular wavenumbers (see $[41,12]$ ). In fact, in some flow cases, the amplification of the external forcing at a given frequency can mostly result from the resonance of a given eigenmode, though other eigenmodes can also contribute to the response, albeit to a lesser extent. In the present case, the main resolvent peak is found for $\omega=7.8$, providing a gain of $R(\omega=7.8) \approx 40$, although harmonic perturbations with slightly larger or smaller $\omega$ are also amplified more than one order of magnitude. Looking at the optimal forcing and responses at the different frequencies corresponding to the several peaks of the resolvent norm, provided in Fig. 22, one can see that the three most amplified harmonic responses ( $\omega=7.44,7.89,8.48$ in the first, third and bottom row) are very similar to the eigenmodes with the same frequencies recovered by stability analysis. This feature is a clear indication of the existence of a quasi-resonance mechanism at those particular frequencies. As shown in Fig. 23a-c-e, all of these responses have main azimuthal wavenumber $m=1$, which corresponds to the peak wavenumber of the main POD modes. At intermediate forcing frequencies, rather different flow structures are found, with main azimuthal wavenumber ranging from $m=1$ to $m=4$ (see Fig. 23 (b-d)), which are however less amplified. A very similar behaviour is found in different cross-sections further downstream. Fig. 21 (b) shows the resolvent norm in the cross-section $x=7.5$ for the same value of $\alpha=$ 9. As before, the resolvent norm peaks at the frequencies of the least stable modes of the corresponding eigenspectrum (not shown), indicating once again a quasi-resonance mechanism. Moreover, the most amplified optimal responses are similar to those found at $x=6.5$, characterized by very low azimuthal wavenumber $(m=1-2$, as for the main 
POD modes) as shown in Fig. 24.

In conclusion, it appears that the local stability analysis can provide some information about the main wavenumbers and structures within the flow when carried out sufficiently close to the rotor. In that sense, those large coherent structures can be approximated by linear modelling, thus enabling - for instance - control design in combination with the information obtained by perusing the localisation of the optimal forcing. We also observe that, in the far wake, stability analysis does not predict the main frequency and spatial content of the flow; this can be related with the development of the wake further downstream, where the main flow structures have broken down. We further analyse the linear model by means of resolvent analysis and show that, if forced appropriately, linearly stable modes can be effectively amplified, and detected in the flow. Therefore, it appears that in convective flows as a wind turbine wake, flow structures arising upstream can determine also the most amplified frequencies downstream. This suggests that the frequency content of the nonlinear forcing $\mathbf{f}$ (which is often assumed being a white-noise) may be a key factor in determining the emerging flow structures in this types of flows.

\section{Conclusions}

Understanding the dynamics and generation of coherent structures in wind turbine wakes is crucial for the design and efficiency improvement of wind farms. In the present paper, we investigate the origin and development of such coherent structures by performing two-dimensional modal and non-modal stability analysis of the turbulent mean flow developing downstream of a wind turbine rotor invested by a laminar, uniform wind. We considered numerical snapshots computed by Large-Eddy-Simulation using the actuator line technique to simulate the rotor, at $R e=6.3 \times 10^{5}$. Proper orthogonal decomposition analysis is applied for detecting the coherent structures developing in the flow. These modes are applied here for benchmarking the linear modelling. More precisely, two-dimensional linear stability and optimal forcing analyses have been applied at different cross-flow planes. The resulting spatial structures at each frequency are compared with the most energetic coherent structures recovered by POD analysis.

In the closest planes to the rotor (3 to 6 diameters), the main POD modes are characterized by rather high values of the temporal and streamwise angular frequency $\alpha=6-10$, and azimuthal wavenumber $m=1$. These structures, mostly located in the root and tip vortices regions, are characterized by slightly different wavenumbers in the inner and in the outer part of the wake and are recovered in the modal stability analysis. Close to the rotor, the unstable modes are mostly located in the outer part of the wake and have a frequency content consistent with that of the most energetic POD modes. The growth rate of these modes decreases while moving far from the rotor, until they become asymptotically stable. In the far wake, these branches identify structures losing resemblance with respect of the identified POD modes, and are characterized by lower streamwise wavenumbers and higher azimuthal wavenumbers. We further explore the spectral content of the POD modes, by considering optimal forcing and response obtained by the resolvent analysis. In particular, we examined a far-wake cross section at the characteristic streamwise frequency of the third POD mode, namely $\alpha=9$, where the mean flow results to be linearly stable. The response gain showed three distinct frequencies corresponding to three barely stable modes. The two linear modes with lower $\omega$ are localised 
at the wake core, wheareas the mode with a higher $\omega$ is mostly localised in the outer layer of the wake, in correspondence with the shear layer. Moreover, the combination of these three linear modes resembles the third POD mode, taken as reference. Optimal forcing analysis shows, therefore, that these asymptotically stable modes can be amplified by more than one order of magnitude by means of a quasi-resonance mechanism, being able to bypass the growth of the most unstable modes recovered in the far wake. This suggests a scenario in which the coherent structures within the wake are mostly originated by modal instability mechanisms close to the rotor, generating waves of selected frequencies able to trigger a high flow response downstream. Note that the application of the linear modeling of the wake at higher Reynolds number would not represent a challenge from the mathematical viewpoint, as far as a proper small-scale turbulence modeling is used. In this regard, the strong separation of scales provides a sufficient solid hypothesis for this type of analysis, although the physical relevance of these linearised models need to be always verified a posteriori by benchmarking the results with the data-driven modal analysis. From the computational viewpoint, LES and possibly stability analysis will be more expensive.

The fact that the development of coherent structures in the rotor's wake appears to be mostly driven by linear mechanisms may pave a way to design efficient means to passively control the wake meandering and/or the wake recovery. Previous studies based on a simplified flow configuration at low Reynolds number have shown that adding a localised control force mimicking the presence of a solid body in flow regions identified by a sensitivity analysis of linear stability modes can consistently modify the wake dynamics. Linear stability eigenmodes, together with their adjoint counterparts are able to provide valuable information on the shape and location of active or passive means to control the spatial structure, recovery rate, and frequency content of the wake behind a wind turbine, provided that the validity of the mean-flow linear model is verified using appropriate data analyses. Such passive control, whose design is based on stability modes and their adjoints, could be achieved by placing solid or compliant bodies downstream of the rotor, mounted on the nacelle of the turbine. Thus, the results of the present paper can potentially open the route to future works where stability-driven passive control of turbine wakes may be used to optimize the power production of wind farms.

\section{Appendix A. Linear analysis of a turbulent mean flow}

In the present work a triple decomposition of the flow field is employed, following reference [38]

$$
\mathbf{u}(\mathbf{x}, t)=\overline{\mathbf{u}}+\widetilde{\mathbf{u}}+\mathbf{u}^{\prime} .
$$

Time average and phase average of a fluctuating quantity $f(\mathbf{x}, t)$ are defined as

$$
\begin{gathered}
\overline{f(\mathbf{x})}=\lim _{T \rightarrow \infty} \int_{t=0}^{t=T} f(\mathbf{x}, t) d t, \\
\langle f(\mathbf{x}, t)\rangle=\lim _{N \rightarrow \infty} \frac{1}{N} \sum_{n=0}^{N} f(\mathbf{x}, t+n \tau),
\end{gathered}
$$

respectively, where $\tau$ is the period of the fluctuation. The wave component $\widetilde{f}$ is then defined as $\widetilde{f}=\langle f\rangle-\bar{f}$. Substituting the triple decomposition into the Navier-Stokes 
equations and taking the time average, the equations for the mean flow are obtained,

$$
\overline{\mathbf{u}} \cdot \boldsymbol{\nabla} \overline{\mathbf{u}}=-\nabla \bar{p}+\nabla \cdot\left(\frac{2}{R e} \overline{\mathbf{S}}-\overline{\widetilde{\mathbf{u}} \widetilde{\mathbf{u}}}-\overline{\mathbf{u}^{\prime} \mathbf{u}^{\prime}}\right) .
$$

The organized wave satisfies the phase-averaged Navier-Stokes equations, after Eq. (A.3) is subtracted

$$
\frac{\partial \widetilde{\mathbf{u}}}{\partial t}+\overline{\mathbf{u}} \cdot \nabla \widetilde{\mathbf{u}}+\widetilde{\mathbf{u}} \cdot \nabla \overline{\mathbf{u}}=-\nabla \widetilde{p}+\nabla \cdot\left(\frac{2}{R e} \widetilde{\mathbf{S}}-\widetilde{\widetilde{\mathbf{u}}}-\widetilde{\mathbf{u}^{\prime} \mathbf{u}^{\prime}}\right)
$$

where $\overline{\mathbf{S}}=\frac{\boldsymbol{\nabla} \overline{\mathbf{u}}+\boldsymbol{\nabla} \overline{\mathbf{u}}^{T}}{2}$ is the mean flow shear stress tensor and $\widetilde{\mathbf{S}}$ the stress tensor of the organized wave. The Reynolds stress tensors $\widetilde{\mathbf{u}^{\prime} \mathbf{u}^{\prime}}$ and $\overline{\mathbf{u}^{\prime} \mathbf{u}^{\prime}}$ are modeled using the Boussinesq hypothesis. Moreover, we assume that the eddy-viscosity field is not oscillating with the perturbation, $\widetilde{\nu}_{t}=0$, and similarly for the turbulent kinetic energy, $\widetilde{k}=0$. With these assumptions, one obtains

$$
\begin{gathered}
\overline{\mathbf{u}^{\prime} \mathbf{u}^{\prime}}=\frac{2}{3} \bar{k} \mathbf{I}-2 \bar{\nu}_{t} \overline{\mathbf{S}}, \\
\widetilde{\mathbf{u}^{\prime} \mathbf{u}^{\prime}}=-2 \bar{\nu}_{t} \widetilde{\mathbf{S}}
\end{gathered}
$$

We refer the interested reader to the work in reference [46] for further details. The eddy viscosity $\bar{\nu}_{t}$ can be determined from equation (A.5a) and used as it is for the oscillating Reynolds stresses (A.5b), similarly to what is done in Newtonian eddy models. As already reported in Eq. (6), we compute $\bar{\nu}_{t}$ as

$$
\bar{\nu}_{t}=-\frac{\overline{\mathbf{u}^{\prime} \mathbf{u}^{\prime}}: \overline{\mathbf{S}}}{2 \overline{\mathbf{S}}: \overline{\mathbf{S}}} .
$$

Appendix B. Resolvent analysis computation

The resolvent analysis is carried out following the approach proposed in reference [41], which is recalled here. As already stated in Eq. (12), we can identify the optimal response of the system due to a forcing at a frequency $\omega$ by maximizing the ratio

$$
R(\omega)=\max _{\hat{\mathbf{f}}} \frac{\left\|(\mathbf{L}-\omega \mathbf{I})^{-1} \hat{\mathbf{f}}\right\|_{E}}{\|\hat{\mathbf{f}}\|_{E}} .
$$

The solution can be obtained by direct or iterative methods, the latter being employed when the computational costs make the solution of the problem prohibitive. Here we employ a direct method. In order to lower the costs of solving Eq. B.1, we consider the 
space $\mathbb{S}^{N}=\operatorname{span}\left\{\tilde{\mathbf{q}}_{\mathbf{1}}, \tilde{\mathbf{q}}_{\mathbf{2}}, \ldots, \tilde{\mathbf{q}}_{\mathbf{N}}\right\}$ spanned by the first $N$ eigenfunctions of $\mathbf{L}$ and expand onto it the vector functions $\mathbf{q}, \mathbf{f} \in \mathbb{S}^{N}$, such that

$$
\mathbf{q}=\sum_{n=1}^{N} \kappa_{n}(t) \tilde{\mathbf{q}}_{\mathbf{n}} \quad \text { and } \quad \mathbf{f}=\sum_{n=1}^{N} \kappa_{n}^{f}(t) \tilde{\mathbf{q}}_{\mathbf{n}} .
$$

The forced problem in Eq. (10) is restated as

$$
\begin{gathered}
\frac{d \kappa}{d t}=\boldsymbol{\Lambda} \kappa+\kappa^{f}(t) \quad \kappa^{f}(t)=\kappa^{f} e^{i \omega t} \\
\kappa=(i \omega \mathbf{I}-\boldsymbol{\Lambda})^{-1} \kappa^{f}
\end{gathered}
$$

with

$$
\begin{gathered}
\kappa=\left(\kappa_{1}, \kappa_{2}, \ldots, \kappa_{N}\right)^{T}, \quad \kappa^{f}=\left(\kappa_{1}^{f}, \kappa_{2}^{f}, \ldots, \kappa_{N}^{f}\right)^{T} \\
\boldsymbol{\Lambda}=\operatorname{diag}\left\{\lambda_{1}, \lambda_{2}, \ldots, \lambda_{N}\right\} .
\end{gathered}
$$

The operator $\Lambda$ represents the linear evolution operator $\mathbf{L}$, projected onto the space $\mathbb{S}^{N}$. The resolvent norm in (B.1) requires the calculation of the energy norm of the state vector $\mathbf{q}$ that can be performed as

$$
\|\hat{\mathbf{q}}\|_{E}=\hat{\mathbf{q}}^{*} \mathbf{M} \hat{\mathbf{q}}=\kappa^{*} \mathbf{M}_{1} \kappa=\|\kappa\|_{E}
$$

where $\hat{\mathbf{q}}=\mathbf{V e} \kappa$ and $\mathbf{V e}$ contains $\mathrm{N}$ eigenvectors of $\mathbf{L}$, while $\mathbf{M}$ and $\mathbf{M}_{\mathbf{1}}$ are suitable energy weight matrices. $\mathbf{M}_{\mathbf{1}}$ is both Hermitian and positive definite, thus we can compute its Cholesky factorization $\mathbf{M}_{1}=\mathbf{F}_{1}^{*} \mathbf{F}_{1}$

$$
\|\kappa\|_{E}=\kappa^{*} \mathbf{F}_{\mathbf{1}}^{*} \mathbf{F}_{\mathbf{1}} \kappa=\left\langle\mathbf{F}_{\mathbf{1}} \kappa, \mathbf{F}_{\mathbf{1}} \kappa\right\rangle=\left\|\mathbf{F}_{\mathbf{1}} \kappa\right\|_{2} .
$$

Using the relations in (B.5)-(B.6) and the equation (10) we rewrite the resolvent norm as

$$
\begin{aligned}
R(\omega) & =\max _{\hat{\mathbf{f}}} \frac{\left\|(i \omega \mathbf{I}-\mathbf{L})^{-1} \hat{\mathbf{f}}\right\|_{E}}{\|\hat{\mathbf{f}}\|_{E}}=\max _{\kappa_{f}} \frac{\left\|\mathbf{F}_{\mathbf{1}}(i \omega \mathbf{I}-\boldsymbol{\Lambda})^{-1} \kappa^{f}\right\|_{2}}{\left\|\mathbf{F}_{\mathbf{1}} \kappa^{f}\right\|_{2}} \\
& =\max _{\kappa_{f}} \frac{\left\|\mathbf{F}_{\mathbf{1}}(i \omega \mathbf{I}-\boldsymbol{\Lambda})^{-1} \mathbf{F}_{\mathbf{1}}{ }^{-1} \mathbf{F}_{\mathbf{1}} \kappa^{f}\right\|_{2}}{\left\|\mathbf{F}_{\mathbf{1}} \kappa^{f}\right\|_{2}}=\left\|\mathbf{F}_{\mathbf{1}}(i \omega \mathbf{I}-\boldsymbol{\Lambda})^{-1} \mathbf{F}_{\mathbf{1}}{ }^{-1}\right\|_{2} .
\end{aligned}
$$

By indicating $\mathbf{B}=\mathbf{F}_{\mathbf{1}}(\boldsymbol{\Lambda}-i \omega \mathbf{I})^{-1} \mathbf{F}_{\mathbf{1}}{ }^{-1}$, the singular values decomposition lead to

$$
\mathbf{B V}=\mathbf{U} \boldsymbol{\Sigma},
$$

where $\mathbf{V}$ and $\mathbf{U}$ are unitary matrices and $\Sigma$ is a diagonal matrix consisting of singular values ordered in size $\sigma_{1} \geq \sigma_{2} \geq \cdots \geq \sigma_{N}$. Concentrating only on the column vectors of $\mathbf{V}$ and $\mathbf{U}$ corresponding to $\sigma_{1}$, which are referred to as the principal right and left singular vectors respectively, one obtains

$$
\mathbf{B} v_{1}=\sigma_{1} u_{1} .
$$

This describes a mapping $\mathbf{B}$ of an input vector $v_{1}$ onto an output vector $u_{1}$ that is also stretched by a factor of $\sigma_{1}$ equal to the 2-norm of $\mathbf{B}$. Therefore, $v_{1}$ describes the most 
responsive disturbance that will be amplified by a factor of $\sigma_{1}=\|\mathbf{B}\|_{2}=R(\omega)$ and $u_{1}$ represents the corresponding response. The optimal forcing and response in spatial coordinates, $\hat{\mathbf{f}}$ and $\hat{\mathbf{q}}$, normalized by their energy norm, are given by

$$
\begin{gathered}
\hat{\mathbf{f}}=\mathbf{V e F}_{\mathbf{1}}^{-\mathbf{1}} \mathbf{F}_{\mathbf{1}} \kappa^{f}=\mathbf{V e F}_{\mathbf{1}}^{-\mathbf{1}} v_{1}, \\
\hat{\mathbf{q}}=\mathbf{V e F}_{\mathbf{1}}^{-\mathbf{1}} \sigma_{1}^{-1} \mathbf{F}_{\mathbf{1}} \kappa=\mathbf{V e F}_{\mathbf{1}}^{-\mathbf{1}} u_{1}
\end{gathered}
$$

\section{References}

[1] International Energy Agency. Renewable 2020, analysis and forecast to 2025. Technical report, Fuel report November 2020, 2020.

[2] International Renewable Energy Agency. Future of wind: Deployment, investment, technology, grid integration and socio-economic aspects (a global energy transformation paper). Technical report, IRENA, 2019.

[3] International Renewable Energy Agency. Renewable capacity statistics 2021. Technical report, IRENA, 2021.

[4] International Renewable Energy Agency. Renewable power generation costs in 2019. Technical report, IRENA, 2021.

[5] R. Ashton, F. Viola, S. Camarri, F. Gallaire, and G. V. Iungo. Hub vortex instability within wind turbine wakes: Effects of wind turbulence, loading conditions, and blade aerodynamics. Phys. Rev. Fluids, 1:073603, Nov 2016.

[6] R Ashton, F Viola, F Gallaire, and G V Iungo. Effects of incoming wind condition and wind turbine aerodynamics on the hub vortex instability. J. Phys. Conf. Ser., 625:012033, jun 2015.

[7] R J Barthelmie, S T Frandsen, M N Nielsen, S C Pryor, P-E Rethore, and H E Jørgensen. Modelling and measurements of power losses and turbulence intensity in wind turbine wakes at middelgrunden offshore wind farm. Wind. Energy, 10(6):517$528,2007$.

[8] D Bastine, L Vollmer, M Wächter, and J Peinke. Stochastic wake modelling based on pod analysis. Energies, 11(3):612, 2018.

[9] D Bastine, B Witha, M Wächter, and J Peinke. Towards a simplified dynamic wake model using pod analysis. Energies, 8(2):895-920, 2015.

[10] G Berkooz, P Holmes, and J L Lumley. The proper orthogonal decomposition in the analysis of turbulent flows. Annu. Rev. Fluid Mech., 25(1):539-575, 1993.

[11] M. A. Bucci. Subcritical and supercritical dynamics of incompressible flow over miniaturized roughness elements. PhD thesis, École Nationale Supérieure d'Arts et Métiers, 2017. 
[12] M. A. Bucci, D. K. Puckert, C. Andriano, J.-Ch. Loiseau, S. Cherubini, J.-Ch. Robinet, and U. Rist. Roughness-induced transition by quasi-resonance of a varicose global mode. J. Fluid Mech., 836:167-191, 2018.

[13] U Ciri, G Petrolo, M V Salvetti, and S Leonardi. Large-eddy simulations of two inline turbines in a wind tunnel with different inflow conditions. Energies, 10(6):821, 2017.

[14] U Ciri, MV Salvetti, K Carrasquillo, C Santoni, GV Iungo, and S Leonardi. Effects of the subgrid-scale modeling in the large-eddy simulations of wind turbines. In Direct and Large-Eddy Simulation X, pages 109-115. Springer, 2018.

[15] G. De Cillis, S. Cherubini, O. Semeraro, S. Leonardi, and P. De Palma. Data driven modal decomposition of the wake behind an nrel-5mw wind turbine. Proceeding of the European Turbulence Conference ETC14, 2021.

[16] G. De Cillis, S. Cherubini, O. Semeraro, S. Leonardi, and P. De Palma. Pod-based analysis of a wind turbine wake under the influence of tower and nacelle. Wind. Energy, 24:609-633, 2021.

[17] M. Debnath, C. Santoni, S. Leonardi, and G. V. Iungo. Towards reduced order modelling for predicting the dynamics of coherent vorticity structures within wind turbine wakes. Philos. Trans. Royal Soc. A, 375, 2017.

[18] J. C. del Álamo and J. Jiménez. Linear energy amplification in turbulent channels. J. Fluid Mech., 559:205-213, 2006.

[19] B. F. Farrell and P. J. Ioannou. Optimal excitation of three-dimensional perturbations in viscous constant shear flow. Phys. Fluids, 1993.

[20] E Ferrer, O Browne, and E Valero. Sensitivity analysis to control the far-wake unsteadiness behind turbines. Energies, 10(10):1599, Oct 2017.

[21] F Giannetti and P Luchini. Structural sensitivity of the first instability of the cylinder wake. J. Fluid Mech., 581:167-197, 2007.

[22] F Gómez, HM Blackburn, M Rudman, AS Sharma, and BJ McKeon. A reducedorder model of three-dimensional unsteady flow in a cavity based on the resolvent operator. J. Fluid Mech., 798, 2016.

[23] Y. Hwang and C. Cossu. Amplification of coherent streaks in the turbulent C,ouette flow: an input-output analysis at low R,eynolds number. J. Fluid Mech., 643:333$348,2010$.

[24] G V Iungo, F Viola, S Camarri, F Porté-Agel, and journal=J. Fluid Mech. volume $=737$ pages $=499-526$ year $=2013$ publisher $=$ Cambridge University Press Gallaire, F. Linear stability analysis of wind turbine wakes performed on wind tunnel measurements.

[25] S Ivanell, R Mikkelsen, Jens N S, and D Henningson. Stability analysis of the tip vortices of a wind turbine. Wind. Energy, 13(8):705-715, 2010. 
[26] P A Krogstad and P E Eriksen. Blind test calculations of the performance and wake development for a model wind turbine. Renew. Energy, 50:325-333, 2013.

[27] L Lesshafft, O Semeraro, V Jaunet, A VG Cavalieri, and P Jordan. Resolvent-based modeling of coherent wave packets in a turbulent jet. Phys. Rev. Fluids, 4(6):063901, 2019 .

[28] M Luhar, AS Sharma, and BJ McKeon. On the structure and origin of pressure fluctuations in wall turbulence: predictions based on the resolvent analysis. J. Fluid Mech., pages 1-33, 2014.

[29] J.L. Lumley. Stochastic tools in turbulence. Applied mathematics and mechanics. Academic Press, 1970.

[30] W. V. R. Malkus. Outline of a theory of turbulent shear flow. J. Fluid Mech., 1:521-539, 1956.

[31] L A Martínez-Tossas, M J Churchfield, and S Leonardi. Large eddy simulations of the flow past wind turbines: actuator line and disk modeling. Wind. Energy, 18(6):1047-1060, 2015.

[32] B. J. McKeon and A. S. Sharma. A critical-layer framework for turbulent pipe flow. J. Fluid Mech., 658:336-382, 2010.

[33] D. Mehta, A.H. van Zuijlen, B. Koren, J.G. Holierhoek, and H. Bijl. Large eddy simulation of wind farm aerodynamics: A review. J. Wind. Eng. Ind. Aerodyn., 133:1-17, 2014.

[34] Pierluigi Morra, Onofrio Semeraro, Dan S. Henningson, and Carlo Cossu. On the relevance of reynolds stresses in resolvent analyses of turbulent wall-bounded flows. J. Fluid Mech., 867:969-984, 2019.

[35] Paolo Orlandi. Fluid flow phenomena: a numerical toolkit, volume 55. Springer Science \& Business Media, 2012.

[36] I. Orlanski. A simple boundary condition for unbounded hyperbolic flows. J. Comput. Phys., 21:251-269, 1976.

[37] C. Picard and J. Delville. Pressure velocity coupling in a subsonic round jet. Int. J. Heat Fluid Fl., 2000.

[38] WC Reynolds and AKMF Hussain. The mechanics of an organized wave in turbulent shear flow. part 3. theoretical models and comparisons with experiments. J. Fluid Mech., 54(2):263-288, 1972.

[39] C Santoni, K Carrasquillo, I Arenas-Navarro, and S Leonardi. Effect of tower and nacelle on the flow past a wind turbine. Wind. Energy, 20:1927-1939, 2017.

[40] S Sarmast, R Dadfar, R F Mikkelsen, P Schlatter, S Ivanell, J N Sørensen, and D S Henningson. Mutual inductance instability of the tip vortices behind a wind turbine. J. Fluid Mech., 755:705-731, 2014. 
[41] P. J. Schmid and D. S. Henningson. Stability and Transition in Shear Flows. Number v. 142 in Applied Mathematical Sciences. Springer-Verlag, 2001.

[42] O T Schmidt, A Towne, G Rigas, T Colonius, and G A Brès. Spectral analysis of jet turbulence. J. Fluid Mech., 855:953-982, 2018.

[43] L Sirovich. Turbulence and the dynamics of coherent structures. parts i-iii. Q. Appl. Math., 45(3):561-590, 1987.

[44] J N Sørensen, R F Mikkelsen, D S Henningson, S Ivanell, S Sarmast, and S J Andersen. Simulation of wind turbine wakes using the actuator line technique. Philos. Trans. Royal Soc. A, 373(2035):20140071, 2015.

[45] R J A M Stevens and C Meneveau. Flow structure and turbulence in wind farms. Annu. Rev. Fluid Mech., 49, 2017.

[46] O. Tammisola and MP. Juniper. Coherent structures in a swirl injector at re $=4800$ by nonlinear simulations and linear global modes. J. Fluid Mech., 792:620-657, 2016.

[47] A Towne, A Lozano-Durán, and X Yang. Resolvent-based estimation of space-time flow statistics. J. Fluid Mech., 883:A17, 2020.

[48] A. Towne, O. T. Schmidt, and T. Colonius. Spectral proper orthogonal decomposition and its relationship to dynamic mode decomposition and resolvent analysis. $J$. Fluid Mech., 847:821-867, 2018.

[49] C VerHulst and C Meneveau. Large eddy simulation study of the kinetic energy entrainment by energetic turbulent flow structures in large wind farms. Phys. Fluids, 26(2):025113, 2014.

[50] L.J. Vermeer, J.N. Sørensen, and A. Crespo. Wind turbine wake aerodynamics. Prog. Aerosp. Sci., 39(6):467-510, 2003.

[51] F Viola, G V Iungo, S Camarri, F Porté-Agel, and F Gallaire. Instability of wind turbine wakes immersed in the atmospheric boundary layer. J. Phys. Conf. Ser., 625:012034, jun 2015.

[52] F. Viola, G. V. Iungo, S. Camarri, F. Porté-Agel, and F. Gallaire. Prediction of the hub vortex instability in a wind turbine wake: stability analysis with eddy-viscosity models calibrated on wind tunnel data. J. Fluid Mech., 750:R1, 2014.

[53] R. Wiser, J. Rand, J. Seel, P. Beiter, E. Baker, E. Lantz, and P. Gilman. Expert elicitation survey predicts $37 \%$ to $49 \%$ declines in wind energy costs by 2050 . Nat. Energy, 2021.

[54] X Yang and F Sotiropoulos. A review on the meandering of wind turbine wakes. Energies, 12(24):4725, Dec 2019. 